\title{
Buffering volatility: Storage investments and technology-specific renewable energy support ${ }^{\dagger}$
}

\author{
Jan Abrella, b, c,*, Sebastian Rausch ${ }^{\text {a, b, d }}$, Clemens Streitberger ${ }^{\text {a, b, d }}$ \\ a Department of Management, Technology, and Economics, ETH Zurich, Switzerland \\ ${ }^{\mathrm{b}}$ Centre for Energy Policy and Economics (CEPE),ETH Zurich,Switzerland \\ ${ }^{c}$ Center for Energy and Environment, Zurich University of Applied Science Winterthur, Switzerland \\ ${ }^{\mathrm{d}}$ Center for Economic Research at ETH Zurich (CER-ETH), Switzerland
}

\section{A R T I C L E I N F O}

\section{Article history:}

Received 10 January 2019

Received in revised form 15 July 2019

Accepted 31 July 2019

Available online xxxx

\section{JEL classification:}

C63

Q42

Q48

Q54

\section{Keywords:}

Renewable energy

Electricity

Volatility

Intermittency

Storage

Technology-specific regulation

Subsidies

Energy policy

Climate policy

\begin{abstract}
A B S T R A C T
Mitigating climate change will require integrating large amounts of highly intermittent renewable energy (RE) sources in future electricity markets. Considerable uncertainties exist about the cost and availability of future large-scale storage to alleviate the potential mismatch between demand and supply. This paper examines the suitability of regulatory (public policy) mechanisms for coping with the volatility induced by intermittent RE sources, using a numerical equilibrium model of a future wholesale electricity market. We find that the optimal RE subsidies are technology-specific reflecting the heterogeneous value for system integration. Differentiated RE subsidies reduce the curtailment of excess production, thereby preventing costly investments in energy storage. Using a simple cost-benefit framework, we show that a smart design of RE support policies significantly reduces the level of optimal storage. We further find that the marginal benefits of storage rapidly decrease for short-term (intra-day) storage and are small for long-term (seasonal) storage independent of the storage level. This suggests that storage is not likely to be the limiting factor for decarbonizing the electricity sector.
\end{abstract}

(c) 2019 Published by Elsevier B.V.

\section{Introduction}

The combat against climate change requires to substantially reduce worldwide carbon dioxide $\left(\mathrm{CO}_{2}\right)$ emissions in the electricity sector over the next decades by profoundly shifting energy supply towards renewable energy (RE) sources. At the global level, the required share of electricity coming from RE sources to restrict global warming to $1.5^{\circ} \mathrm{C}$ is estimated to be between $70 \%$ and $81 \%$

\footnotetext{
is We gratefully acknowledge financial support by the Swiss Competence Center for Energy Research, Competence Center for Research in Energy, Society and Transition (SCCER-CREST) and Innosuisse (Swiss Innovation Agency).

* Corresponding author at: Center for Energy and Environment, ZHAW Winterthur, Switzerland.

E-mail addresses: abre@zhaw.ch (J. Abrell), srausch@ethz.ch (S. Rausch), clemenss@ethz.ch (C. Streitberger).
}

by 2050 (IPCC, 2018). For Europe, the European Commission's (2011) Energy Roadmap 2050 foresees RE shares as high as 64\% to $97 \%$ to be consistent with EU climate policy targets. Such high amounts of energy supplied from RE sources pose significant challenges to existing energy systems as the economically most viable and carbonfree RE technologies (i.e., wind and solar) are highly volatile in their output.

Fig. 1 shows the temporal variation of electricity demand and resource availability of wind and solar over the course of a day (Panel a) and a year (Panel b). It serves to illustrate the well-known and fundamental issue which also motivates our analysis: a future low-carbon energy system which relies on a large share of volatile RE energy will likely face the challenge of substantial periodic mismatches between energy demand and supply. To cope with the high volatility of daily and seasonal resource availability, a mechanism is needed to shift supply between hours of the day and possibly between 


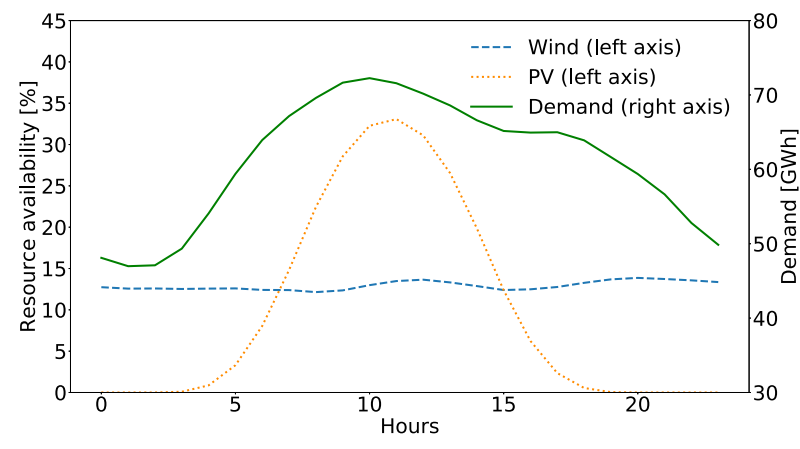

(a) By hour over a day.

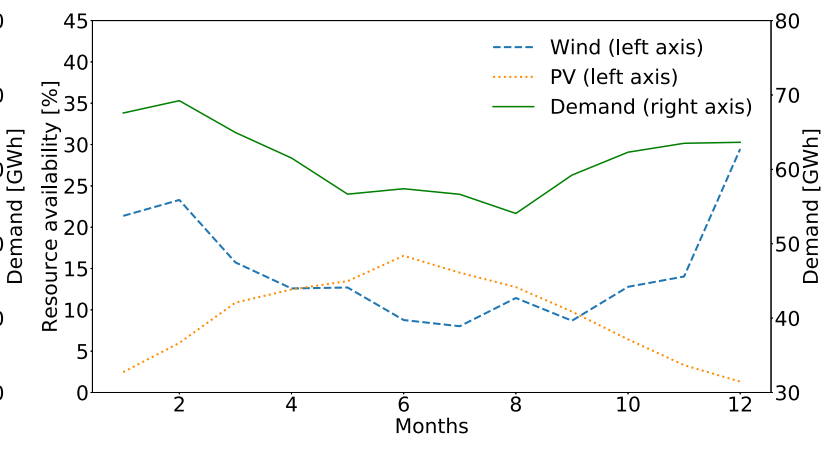

(b) By month over a year.

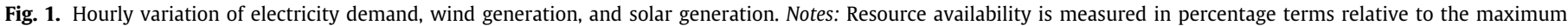

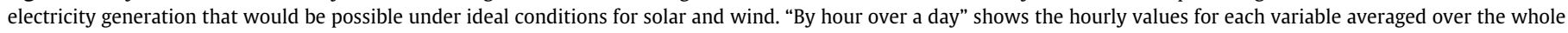

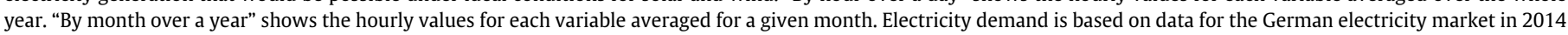

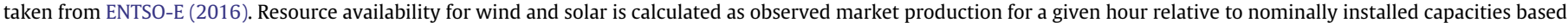
on data from German transmission system operators (50Hertz, 2018; Amprion, 2018; Tennet, 2018; TransnetBW, 2018).

seasons (for example, by either shifting solar generation from day to night or from summer to winter, or wind generation from off-peak to peak hours). ${ }^{1}$

Much of the academic literature and ongoing discussions among policymakers have focused on the question how energy storage can serve as a buffering mechanism to cope with the volatility and system integration costs induced by intermittent RE sources (Hirth, 2015; Gowrisankaran et al., 2016; Sinn, 2017; Zerrahn et al., 2018). At the same time, there are considerable uncertainties as well as concerns about the costs, availability, and potentials of future storage technologies, in particular when deployed at the large scales required for deep decarbonization. ${ }^{2}$

Instead of focusing on a pure technological solution for buffering volatility (i.e., through energy storage), this paper examines the suitability of a regulatory or public policy mechanism as a means for coping with the impacts of large shares of highly volatile RE sources in future energy systems: the design of technology-specific RE support schemes. Specifically, we ask to what extent the economic cost of integrating a large amount of highly volatile wind and solar energy can be reduced by modifying the design of RE support schemes-such as subsidies on output or investment-to take into account the heterogeneous value of different RE technologies with respect to system integration costs. Current policy approaches tend to favor technologyneutral support schemes. A recent example are German joint tenders for wind and solar energy, the last of which saw a dominance of solar bids over wind (BNetzA, 2019). In contrast to such policy designs our fundamental proposal is to improve existing energy market regulation in a way which exploits the complementarities of wind and solar technologies in terms of their underlying heterogeneous resource profiles and the correlation with time-varying electricity demand.

\footnotetext{
1 The profile of solar largely coincides with the demand peak around midday; during nighttime, however, demand is still large (although being at its lowest level), while solar generation is zero. The correlation coefficient between demand and solar availability is 0.48 . In contrast, wind shows a relatively flat availability pattern, implying an advantage during night hours when there is no generation from solar. At the same time, however, wind is ill-suited to meet demand over the day, in particular during peak hours. The correlation coefficient for wind is 0.23 . Over the course of a year, seasonal changes in the monthly average of demand and resource availabilities show a different picture: solar generation is negatively correlated with demand (with a coefficient of -0.74 ) whereas wind closely follows demand exhibiting a strong positive correlation (with a coefficient of 0.72 ).

2 As of today, the only energy storage technology for electricity used at large scale is hydroelectric pumped-storage power (Schwab, 2009), representing about 99\% of the worldwide installed storage capacity (Rastler, 2010).
}

We also investigate how the need for energy storage changes when this alternative buffering mechanism is optimally exploited. To the best of our knowledge, we are the first to examine the potential role of policy design for reducing the cost of integrating volatile RE supply.

To provide a conceptual and empirically-grounded framework for thinking about the economics of integrating high shares of volatile RE sources into an electricity market, we develop a numerical partial equilibrium model of a wholesale electricity market which resolves output decisions on hourly markets, time-dependent demand and resource availabilities of wind and the sun, investments in RES production capacity, curtailment decisions to maintain system stability, and a detailed representation of the functioning of electricity storage. Storage capacity is varied exogenously ${ }^{3}$ in order to gauge its impact on overall system integration cost of renewables. The decentralized market model is embedded in a welfare-maximizing problem of a benevolent regulator who chooses RE support policies (through subsidies on RE output which we model as a feed-in premium on top of the market price) in order to implement an electricity market with a high share of intermittent RE at the lowest cost to society. While we calibrate the model to stylized conditions of the German electricity market, we think that the main insights from our analysis are also relevant for the electricity market context of many other countries.

Our analysis provides several important insights. First, we find that the storage capacity needed to accommodate high shares of intermittent RE output is relatively moderate, even under a technology-neutral RE support scheme. This implies that the potentially high costs of providing storage at large scale in the future need not jeopardize the achievement of environmental targets (i.e., the reduction of $\mathrm{CO}_{2}$ emissions through increasing the share of low-carbon renewables). Second, we find that the design of a RE support policy can have a significant impact on system integration cost as well as storage capacity needs when there are several intermittent renewable technologies with heterogeneous availability patterns of the underlying natural resources (such as wind and solar energy). The smart differentiation of RE subsidies affects investment patterns in a way which can effectively reduce the curtailment of excess generation, in turn lowering the need for costly investment in energy storage. We use a simple

\footnotetext{
3 In other words, agents in the model make decisions on electricity dispatch and investment into RE capacity for several given storage levels without taking investment cost for storage into consideration. Storage cost is then accounted for in an ex-post cost-benefit analysis with cost estimates for current storage technologies.
} 
cost-benefit framework to show that optimal subsidy differentiation significantly reduces the level of optimal storage. In this sense, concerns about the costs and availability of storage technologies in order to enable the integration of high shares of intermittent RE supply in future electricity markets and to achieve environmental goals are even more diminished if a smart design of RE support policies is chosen. Third, within our modeling framework which captures high RE shares up to $80 \%$ but not a completely decarbonized system, we find that the type of storage most likely needed is short-term to medium-term storage. The additional benefits from long-term seasonal storage are relatively modest and most likely much smaller than its investment costs.

This paper contributes to the existing literature in several ways. First, we add to the main insight, supported by a growing body of economic and technical studies (see, for example, Zerrahn et al., 2018, and references therein), that in order to integrate large shares of volatile RE supply in future energy systems only moderate levels of energy storage are needed.

Second, there is a growing literature on storage capacity in electricity markets and its connection to the expanding renewable generation capacities. Linn and Shih (2016) investigate the impact of the introduction of large storage capacities into current electricity systems using numerical modeling of the Texas ERCOT region and stylized theoretical considerations to assess the impact on total carbon emissions of a system with dirty base load producers (coal), cleaner peak load producers (gas), and renewables (wind, solar). Carson and Novan (2013) use a theoretical model and empirical methods to show the same effect in the ERCOT region, and, in addition, an adverse impact of increased storage capacities on renewables with high production correlation to peak demand (solar) and a positive impact on renewables which produce at base-load hours (wind) due to a price-leveling effect of storage. Crampes and Moreaux (2010) use a theoretical model of a hydro pumped-storage operator and a fossil generator to determine optimal joint usage of both technologies; they do not consider intermittent RE sources. Helm and Mier (2018) examine the effect on $\mathrm{CO}_{2}$ emissions of subsidizing energy storage. In contrast to the abovementioned papers, we focus on market conditions as we expect in a future electricity market with a very high level of intermittent RE supply and highlight the role of regulatory design, besides energy storage, for buffering volatility.

Third, we also make a connection to the emerging literature investigating the consequences of the fundamental heterogeneity of RE technologies with respect to availability patterns. Abrell et al. (2019b) consider the environmental value and market value of different renewables and define an environmental motive for differentiating subsidies by technology, while Fell and Linn (2013) and Wibulpolprasert (2016) investigate the impact of resource heterogeneity on cost-effectiveness of different abatement policies. Empirical studies like Abrell et al. (2019a) evaluate different market values and environmental values of RE sources ex-post. While these studies highlight the need for improved policy design to incorporate external effects at the system or market level, they focus on $\mathrm{CO}_{2}$ emissions but abstract from storage investments and the issue of the cost of integrating volatile RE supply for decarbonizing the electricity sector.

The remainder of this paper is structured as follows. Section 2 presents the electricity market model. Section 3 provides detail about the empirical specification of the model (against the context of the German electricity market). Section 4 presents the main findings from the simulations investigating the trade-offs between storage capacity and the role of technology-differentiated RE support policy as potential buffering mechanisms. Section 5 presents a simple cost-benefit analysis to gauge the level of optimal energy storage needed to implement a market with a high share of volatile RE supply under different assumptions about RE policy design. Section 7 concludes by discussing implications and caveats of our analysis.

\section{The model}

To assess alternative strategies for integrating a large share of intermittent RE into an energy system, we employ a partial equilibrium model of the wholesale electricity market which resolves output decisions on hourly markets, time-dependent demand and resource availabilities of wind and sun, investment decisions in production capacity, curtailment decisions to maintain system stability, and the functioning of electricity storage. The decentralized equilibrium model is embedded in a welfare-maximizing problem of a benevolent regulator who aims to implement an electricity market with a high share of intermittent RE at lowest cost.

\subsection{The regulator's problem}

The model comprises two levels. At the top level, a benevolent regulator is concerned with the problem of implementing an exogenous and given minimum level of RE generation in the market at the lowest attainable total system cost $\mathscr{C}$ to society. The choice variable is a RE support scheme which can take on the form of either a technology-neutral support or technology-differentiated support. In implementing the RE support scheme, the regulator has to take into account the equilibrium conditions of the electricity market. Formally, the regulator's problem is then given by:

$$
\begin{aligned}
& \min _{\boldsymbol{b}} \mathscr{C}(\boldsymbol{P}(\boldsymbol{b}), \boldsymbol{X}(\boldsymbol{b})) \\
& \text { s.t. } \boldsymbol{P}(\boldsymbol{b}), \boldsymbol{X}(\boldsymbol{b}) \in \mathscr{E},
\end{aligned}
$$

where $\boldsymbol{b}$ denotes the policy choice of the regulator, $\boldsymbol{P}(\boldsymbol{b}), \boldsymbol{X}(\boldsymbol{b})$ are the prices and quantities constituting the market equilibrium in the electricity market for a given choice of the regulator, and $\mathscr{E}$ is the set of all feasible equilibrium allocations in the wholesale electricity market.

\subsection{Feasible equilibrium allocations $\mathscr{E}$ of the wholesale electricity market}

We formulate the equilibrium conditions of the wholesale electricity market as a mixed complementarity problem (MCP, see Mathiesen, 1985; Rutherford, 1995) which is cast as a system of inequalities which derive from the decision problems of profit-maximizing agents with two types of conditions: zero-profit conditions that are complimentary to quantity variables $\boldsymbol{X}$ and market-clearing conditions complementary to price variables $\boldsymbol{P}$. The economic agents in our model are electricity suppliers which produce either from renewable or from conventional sources. Production technologies are denoted by $i \in \mathscr{I}$ with subsets $\mathscr{G}$ for renewable technologies and $\mathscr{B}$ for conventionals. We indicate time periods by $t \in \mathscr{T}$.

\subsubsection{Energy supply and investment}

Agents maximize their profits by choosing investments $I_{i}$ and generation for each time period $X_{i t}$. The profits are given by

$\Pi_{i}=\sum_{t}\left[\left(P_{t}+\omega_{i} S\right) X_{i t}-c_{i}^{\mathrm{g}}\left(X_{i t}\right)\right]-c_{i}^{\mathrm{i}}\left(I_{i}\right)$,

where $P_{t}$ denotes the market price at time $t, S$ is the RE subsidy per MWh produced which firms receive and $\omega_{i}$ is a policy choice variable for the regulator which allows to differentiate the subsidy by technology if $\omega_{i} \neq \omega_{j}$. For conventional technologies, $i \in \mathscr{B}, \omega_{i}=0$. 
The functions $c_{i}^{\mathrm{g}}\left(X_{i t}\right)$ and $c_{i}^{\mathrm{i}}\left(I_{i}\right)$ denote generation cost and investment cost, respectively.

Output can never exceed installed capacity, so the following condition needs to be fulfilled:

$\alpha_{i t}\left(\bar{k}_{i}+I_{i}\right) \geq X_{i t} \quad \forall i, t$.

The parameter $\alpha_{i t}$ denotes the fraction of available capacity of technology $i$ at time $t$, which captures downtime of conventional generators due to, for example, maintenance and the time-varying availability of renewable technologies (intermittency). $\bar{k}_{i}$ denotes already installed capacity.

For an agent who maximizes profits (Eq. (2)) subject to the capacity constraint (Eq. (3)), we obtain the following first order conditions (FOCs):

$\frac{\partial c_{i}^{\mathrm{g}}\left(X_{i t}\right)}{\partial X_{i t}}+P_{i t}^{\mathrm{I}} \geq P_{t}+\omega_{i} S \quad \perp \quad X_{i t} \geq 0 \quad \forall i, t$

$\frac{\partial c_{i}^{\mathrm{i}}\left(I_{i}\right)}{\partial I_{i}} \geq \sum_{t} \alpha_{i t} P_{i t}^{\mathrm{I}} \quad \perp \quad I_{i} \geq 0 \quad \forall i$

$\alpha_{i t}\left(\bar{k}_{i}+I_{i}\right) \geq X_{i t} \quad \perp \quad P_{i t}^{\mathrm{I}} \geq 0 \quad \forall i, t$.

$P_{i t}^{I}$ is the shadow value of capacity which is complementary to Eq. (3), which is expressed by the perpendicular operator $\perp$. The perpendicular operator indicates that in equilibrium a variable is non-zero when the associated condition holds with equality, whereas it has to be zero when the condition is a strict inequality.

\subsubsection{Storage}

The storage operator maximizes profits $\Pi_{S}$ from selling (release from storage) and buying (injection into storage) electricity. The profit function is given by:

$\Pi_{\Sigma}=\sum_{t}\left(P_{t} R_{t}-P_{t} J_{t}\right)$

where $R_{t}$ denotes release from storage and $J_{t}$ injection into storage. We distinguish three types of capacities which are needed for the storage process: release capacity $\bar{k}^{\mathrm{R}}$, injection capacity $\bar{k}^{\mathrm{J}}$, and storage capacity $\bar{k}^{\Sigma}$. Similar to production, the installed storage capacities constitute constraints to the profit maximization problem of the storage operator, which can be characterized by the following FOCs:

$$
\begin{array}{ll}
M_{t}+P_{t}^{\Sigma} \geq M_{t+1} & \perp \quad \Sigma_{t} \geq 0 \quad \forall t \\
P_{t} \geq \psi M_{t}-P_{t}^{J} & \perp \quad J_{t} \geq 0 \quad \forall t \\
M_{t}+P_{t}^{\mathrm{R}} \geq P_{t} & \perp \quad R_{t} \geq 0 \quad \forall t \\
\bar{k}^{\Sigma} \geq \Sigma_{t} \quad \perp \quad P_{t}^{\Sigma} \geq 0 \quad \forall t \\
\bar{k}^{\mathrm{J}} \geq J_{t} \quad \perp \quad P_{t}^{\mathrm{J}} \geq 0 \quad \forall t \\
\bar{k}^{\mathrm{R}} \geq R_{t} \quad \perp \quad P_{t}^{\mathrm{R}} \geq 0 \quad \forall t .
\end{array}
$$

$P_{t}^{\Sigma}, P_{t}^{\mathrm{J}}$, and $P_{t}^{\mathrm{R}}$ are the shadow values of storage capacity, injection capacity, and release capacity, respectively. The storage efficiency parameter $\psi$ captures roundtrip losses of the storage cycle and $M_{t}$ is the shadow value associated with the following condition which ensures time consistency of storage across periods:

$$
\Sigma_{t}+\psi J_{t}-R_{t}=\Sigma_{t+1} \quad \perp \quad M_{t} \text { free } \quad \forall t .
$$

\subsubsection{Curtailment}

For the curtailment $C_{i t}$ of excess RE generation, we model a system operator who is bound by the RE policy to buy all generation from RE producers paying the market price and a subsidy $P_{t}+\omega_{i} S$ and then sells the electricity in the market at market price $P_{t}$. They choose how much of RE generation to curtail to maintain system stability. Thus, the system operator maximizes the following profit function with choice variable $C_{i t}$ :

$\Pi_{s y s}=\sum_{i, t}\left[P_{t}\left(X_{i t}-C_{i t}\right)-\left(P_{t}+\omega_{i} S\right) X_{i t}\right]$,

under the condition that curtailment cannot exceed production in any period. This leads to the following FOCs:

$P_{t}+P_{i t}^{C} \geq 0 \quad \perp \quad C_{i t} \geq 0 \quad \forall i, t$

$X_{i t} \geq C_{i t} \quad \perp \quad P_{i t}^{C} \geq 0 \quad \forall i, t$,

where $P_{i t}^{C}$ denotes the shadow value of curtailment. ${ }^{4}$

\subsubsection{Market clearing and electricity price}

At any time $t$ electricity demand $\bar{d}_{t}$ needs to be fulfilled. This is expressed by the market clearing condition which is associated with the electricity price $P_{t}:{ }^{5}$

$\sum_{i}\left(X_{i t}-C_{i t}\right)+R_{t}-J_{t}=\bar{d}_{t} \quad \perp \quad P_{t} \quad$ free $\quad \forall t$,

where generation net of curtailment plus release from storage minus injection into storage equals demand.

\subsubsection{RE support}

The regulator's policy choice $\boldsymbol{b}=\left\{\omega_{i}\right\}_{i \in \mathscr{I}}$ concerns the relative subsidy for different renewable technologies $\omega_{i} S$ in Eq. (4). The overall level of the subsidy is determined by the exogenous target $\gamma$ for the share of RE generation in total production. Even though, demand remains inelastic total production changes with increasing use of storage capacity because a part of the generation going into storage, $(1-\psi) J_{t}$, is lost over the storage cycle. Thus, we introduce the following condition to the MCP problem to capture the notion that a given percentage of production over all technologies needs to originate from RE sources:

$$
\sum_{i \in \mathscr{G}, t}\left(X_{i t}-C_{i t}\right) \geq \gamma \sum_{i, t}\left(X_{i t}-C_{i t}\right) \quad \perp \quad S \geq 0,
$$

which formalizes the notion that renewable generation net of curtailment needs to reach a given share $\gamma$ of total net generation.

\subsubsection{Definition of equilibrium}

The set of feasible equilibrium allocations $\mathscr{E}$ is defined by prices and quantities $\{\boldsymbol{p}(\boldsymbol{b}), \boldsymbol{x}(\boldsymbol{b})\}$ with prices $\boldsymbol{p}(\boldsymbol{b})=\left\{P_{t}, P_{i t}^{\mathrm{I}}, P_{t}^{\Sigma}, P_{t}^{\mathrm{J}}, P_{t}^{\mathrm{R}}, P_{i t}^{\mathrm{C}}, M_{t}\right\}$ determined by market-clearing conditions (18), (6), (11), (12), (13), (17), and (14) and quantities $\boldsymbol{x}(\boldsymbol{b})=\left\{X_{i t}, C_{i t}, I_{i}, \Sigma_{t}, J_{t}, R_{t}\right\}$ determined by zero-profit conditions (4), (16), (5), (8), (9), and (10).

\footnotetext{
4 Note that $\bar{k}^{\Sigma}, \bar{k}^{\mathrm{J}}$, and $\bar{k}^{\mathrm{R}}$ are parameters, i.e. we do not model investment decisions in energy storage but rather the problem of how to optimally operate a given storage capacity. Section 5 then turns to the broader problem of choosing an optimal level of storage capacity given associated costs and benefits.

5 The market clearing condition holds with strict equality which implies that its dual variable is free in sign. We continue to use the perpendicular operator $\perp$ to indicate the complementarity $\left[\sum_{i}\left(X_{i t}-C_{i t}\right)+R_{t}-J_{t}-\bar{d}_{t}\right] P_{t}=0$.
} 


\subsection{Total system cost $\mathscr{C}$ and system integration cost}

Total system cost $\mathscr{C}$ is defined by the sum of investment cost for RES and generation cost:

$\mathscr{C}=\sum_{i} c_{i}^{\mathrm{i}}\left(I_{i}\right)+\sum_{i, t} c_{i t}^{\mathrm{g}}\left(X_{i t}\right)$

We now turn to a discussion how we measure system integration cost of intermittent renewables within our model. Generally, system integration cost comprises uncertainty cost, grid expansion cost, and intermittency cost. As intermittency cost is found to make up the largest share in total integration cost (see Hirth et al., 2015; Hirth, 2015; Gowrisankaran et al., 2016), our model abstracts from stochastic weather changes and associated forecast errors and from modeling the electric power grid.

Intermittency cost, i.e. the cost associated with foreseeable variations in resource availability over time, manifests itself in the model as investment inefficiency of RE capacity. The RE target $\gamma$ demands a certain percentage of total consumption of electricity from RE sources but their availabilities, $\left\{\alpha_{i t}\right\}_{t \in \mathscr{T}}$ for $i \in \mathscr{G}$, prohibit them from flexibly satisfying demand $\bar{d}_{t}$ in each period. If $\gamma$ is high, generation from hours with high availability does not suffice to fulfill the overall target. Consequently, investments need to be chosen such that RE capacity contributes, also in hours with low resource availability, substantial amounts of electricity to satisfy demand. In hours with high resource availability, RE generation exceeds demand and the excess generation needs to be shed according to condition (16).

This mechanism thus links curtailment $C_{i t}$ to intermittency cost: the more inefficient the investment and the more total curtailment, the higher the intermittency cost. Our measure for intermittency cost precisely exploits this mechanism by focusing on average investment cost. We calculate investment cost, $c_{i}^{\mathrm{i}}\left(I_{i}\right)$, per net generation, i.e. RE generation net of curtailment, $\sum_{t}\left(X_{i t}-C_{i t}\right)$ :

$\kappa_{i}=\frac{c_{i}^{\mathrm{i}}\left(I_{i}\right)}{\sum_{t}\left(X_{i t}-C_{i t}\right)} \quad \forall i \in \mathscr{G}$

$\kappa_{i}$ measures the efficiency of RE capacity use and is never zero as long as there is investment into RE capacity. As an average value, $\kappa_{i}$ is also useful in comparing system integration cost across situations with different levels of storage investment.

\section{Data and model calibration}

This section describes the data sources used for the calibration of the model presented in Section 2. To calibrate the model we need to specify the following parameters: hourly demand $\bar{d}_{t}$, the timevarying availability factors for RE $\alpha_{i t}$, the efficiency parameters and capacities for storage $\psi, \bar{k}^{\Sigma}, \bar{k}^{\mathrm{J}}$, and $\bar{k}^{\mathrm{R}}$. We also need to choose the functional forms of the cost functions for generation and investment, $c_{i}^{\mathrm{g}}$ and $c_{i}^{\mathrm{i}}$, and estimate their functional parameters based on available data.

For the calibration, an important question regarding the time perspective arises: on the one hand, we want to model the dispatch decisions of economic agents in a market with a very high share of intermittent renewables as is expected to be the situation in a future electricity market (for example, in the year 2050) in which energy regulation steps in to meet strict emission goals; on the other hand, the investment decisions we model need to be taken well before 2050 under market conditions which resemble more closely the current state of the energy system. In order to obtain a stylized and yet fairly realistic representation of RE investment, we thus calibrate the model to the current (i.e., year 2014) conditions of the German electricity market. Since conventional capacity is usually long-lived, we use the current technology mix as a basis for the calibration of the conventional supply curve but use fuel prices in line with predictions for 2050 which would govern future electricity market dispatch decisions.

\subsection{Demand and RE resource availability}

In order to capture the seasonal variation of demand and resource availability of RE technologies, we model an entire year with hourly time resolution. To keep the model numerically tractable we restrict the total number of hours modeled to 8 weeks ( $1344 \mathrm{~h}$ ) which are chosen to represent all four seasons of the year. We take hourly demand $\bar{d}_{t}$ from the European Network of Transmission System Operators (ENTSO-E, 2016). To obtain the availability of RE sources $\alpha_{i t}$ we assume that wind and solar having very low variable production cost will produce electricity whenever the natural resource is available. The fraction of actual production at any given hour and the nominally installed capacity provides us then with a percentage value of resource availability. For this, we use generation data of renewables from German transmission system operators (50Hertz, 2018; Amprion, 2018; Tennet, 2018; TransnetBW, 2018).

\subsection{Storage}

Given the considerable uncertainties about which storage technologies will dominate in the future and about their cost and technical limitations we aim to keep our model framework as flexible as possible for storage. The model setup with the storage Eqs. (8)-(14) is generic in the sense that any storage technology will have some kind of capacity for injection, storage, and release of electricity and time consistency will always have to be guaranteed. ${ }^{6}$ For the capacities associated with storage (storage capacity $\bar{k}^{\Sigma}$, injection capacity $\bar{k}^{J}$, and release capacity $\bar{k}^{\mathrm{R}}$ ), we use the values reported by Hartmann et al. (2012) to specify the storage capacity of the reference case. In the numerical simulation, we vary these values exogenously, that is economic agents do not make endogenous investment decisions for storage capacity but rather treat it as given. Finally, we adopt a $75 \%$ roundtrip efficiency for storage, $\psi$, which is in line with values from the literature for pumped hydro storage (PHS) (see, e.g. Egerer et al., 2014; Newbery, 2016). In their Table B1, Zakeri and Syri (2015) report a range of $70 \%-82 \%$ for PHS and a larger range (60\% to $95 \%$ ) for other technologies, such as compressed air energy storage (CAES) and different kinds of batteries. We will perform a sensitivity analysis for $\psi=60 \%$ and $\psi=90 \%$ to capture the impact of storage efficiency of different technologies on the results (see Section 6).

\subsection{Conventional generation}

We aggregate all fossil-based generation (gas, coal, oil) into one conventional supply curve. We start out by constructing a merit order curve for German power stations with data from Open Power System Data (2017). Electricity generating plants are ranked by marginal production cost taking into account fuel cost and heat efficiencies. Estimates for future fuel prices are taken from IEA's World Energy Outlook (International Energy Agency, 2018). We then fit $\partial c^{\mathrm{g}} / \partial X_{i t}$ as a linear marginal cost curve to the data, which accounts for the rising marginal cost of a heterogeneous fleet of power plants. We report the coefficients of the estimate in Table 1. The original data of the merit order curve and the linear fit are shown in Fig. 2.

We assume that the existing conventional generation capacity is large enough (similar to the current situation in Germany) so as to be

\footnotetext{
6 We can even include demand side management in such a framework in that it is understood as shifting load over time just as physical storage technologies do.
} 
Table 1

Production capacities $\bar{k}_{i}$ and OLS-fitted linear functions for marginal generation cost $\partial c_{i}^{\mathrm{g}} / \partial X_{i t}$ and marginal investment cost $\partial c_{i}^{\mathrm{i}} / \partial I_{i}$.

\begin{tabular}{|c|c|c|c|c|c|c|}
\hline & \multicolumn{3}{|c|}{ Energy supply technologies } & \multicolumn{3}{|c|}{ Electricity storage } \\
\hline & Conventional & Wind & Solar & Storage & Injection & Release \\
\hline \multicolumn{7}{|c|}{ Installed capacities $\left(\bar{k}_{i}, \bar{k}^{\Sigma}, \bar{k}^{\mathrm{J}}, \bar{k}^{\mathrm{R}}\right)$} \\
\hline MW & 90000 & 0 & 0 & - & 6400 & 6400 \\
\hline MWh & - & - & - & 37700 & - & - \\
\hline \multicolumn{7}{|c|}{ Marginal generation cost functions $\left(\partial c_{i}^{\mathrm{g}}\left(X_{i t}\right) / \partial X_{i t}\right)$} \\
\hline Intercept $\left(\frac{€}{\mathrm{MWh}}\right)$ & 5.0 & 0 & 0 & - & - & - \\
\hline Slope $\left(\frac{€}{\mathrm{MWh}^{2}}\right)$ & $2.2 \times 10^{-3}$ & - & - & - & - & - \\
\hline \multicolumn{7}{|c|}{ Marginal investment cost functions $\left(\partial c_{i}^{\mathrm{i}}\left(I_{i}\right) / \partial I_{i}\right)$} \\
\hline Intercept $\left(\frac{€}{\mathrm{MW}}\right)$ & - & 60618 & 41752 & - & - & - \\
\hline Slope $\left(\frac{€}{\mathrm{MW}^{2}}\right)$ & - & 0.24 & 0.06 & - & - & - \\
\hline
\end{tabular}

able to fulfill demand at any time; see Table 1 for the numerical value of $\bar{k}_{\text {conventional }}$. This is tantamount to abstracting from investment decisions in the generation capacity of conventional technologies (i.e., $I_{i}=0$ for $i \in \mathscr{B}$ ).

\subsection{Renewable generation}

Renewable generators incur zero variable generation cost and hence we assume $c_{i}^{\mathrm{g}}\left(X_{i t}\right)=0$ for $i \in \mathscr{G}$. The most important cost parameter for RE sources is investment $\operatorname{cost} c_{i}^{\mathrm{i}}\left(I_{i}\right)$. Since wind and solar energy depend on a natural resource, there is geographical heterogeneity of site quality for installations. Assuming that investments are made in favorable sites first and then continue in locations with decreasing wind and solar resources we model investment cost to be increasing in total investment $I_{i}$ even though nominal investment cost per MW capacity is constant ${ }^{7}$. Thus, we choose a linear functional form for marginal investment $\operatorname{cost} \partial c^{i} / \partial I_{i}$. To estimate the parameters of this function we use data on full load hours ${ }^{8}$ and total capacity potential for each German state from Agentur für Erneuerbare Energien (AEE, 2018) to construct a curve showing resource quality vs. investment into capacity. When starting with the potential with the highest full load hours and continuing in decreasing order the resulting curve is also decreasing in $I_{i}$. We obtain the investment cost curve by dividing nominal annualized investment cost per MW from Kost et al. (2013) by full load hours. The final investment cost curve obtained in this way is increasing in $I_{i}$ and we report the estimated parameters in Table $1 .^{9}$

We adopt a green field approach for RE, that is pre-installed renewable capacity is zero (i.e., $\bar{k}_{i}=0$ for $i \in \mathscr{I}$ ). Investors choose the amount of investment $I_{i}$ according to zero-profit condition (5) and the RE target (19).

\subsection{Computational strategy}

We conclude this section with a short description of the numerical solving strategy that we employ in the simulations. The top-level problem of the regulator, the cost minimization in Eq. (1), is formulated as a Mathematical Program under Equilibrium Constraints

\footnotetext{
7 For our analysis, we abstract from technological progress and a connected positive learning externality. While this would have an impact on the level of the optimal RE subsidy, the motivation for subsidy differentiation to reduce integration cost would be unchanged.

8 Full load hours are a measure for the resource quality at a given site. They translate the total production over a year from a RE generator into the number of hours needed to generate the same amount of electricity at fully employed installed capacity.

9 See Abrell et al. (2019b) for a more detailed description of the calibration method of the investment curves.
}

(MPEC), that is cost is minimized subject to constraints stemming from an equilibrium problem (Luo et al., 1996) which we denoted by the set of feasible allocations $\mathscr{E}$ in Section 2. We express the lowerlevel equilibrium problem as a mixed complementarity problem (MCP) (Mathiesen, 1985; Rutherford, 1995). Due to the lack of robust solvers for MPECs (Luo et al., 1996) we solve the lower level MCP problem over a suitable grid to find the minimum cost and thus the solution to the MPEC using the PATH solver (Dirkse and Ferris, 1995) for complementarity problems and the General Algebraic Modeling System (GAMS).

\section{Buffering volatility: storage investments vs. differentiated renewable energy support}

This section presents the results of our numerical simulations. First we briefly explain the scenarios considered and the simulations that we performed. We continue by summarizing the main findings and then go on to explain the underlying market mechanisms in more detail in the remaining subsections.

\subsection{Design of counterfactual experiments}

To examine the role of the storage investments and differentiated renewable support schemes, we model the following three scenarios:

- No policy assumes that (i) RE support policy is absent and that (ii) storage capacity is equal to the currently installed pumped hydro storage capacity in Germany (37.7 GWh as of 2014). This scenario serves as a suitable reference point for analyzing the additional costs and benefits of future expansions of storage.

- Neutral subsidy assumes that the RE target is implemented by a technology-neutral subsidy (modeled as a market premium) for RE generation.

- Differentiated subsidy assumes that the RE target is implemented by a market premium which is optimally differentiated by RE technology so as to minimize total system cost. ${ }^{10}$

Under No policy, generation from RE makes up $42 \%$ of total generation, which can be broken down further into $22.5 \%$ generation from solar and $19.5 \%$ from wind. Since the RE share in this scenario

\footnotetext{
10 Note that by design investors receive payments for possible generation even if the system operator curtails parts of the RE generation. Thus, investment decisions in RE capacity do not take into account curtailed energy. It is beyond the scope of the paper to study to what extent other policy design, for example, RE subsidies based on installed capacity (i.e., MW instead of MWh), can address integration costs in terms of curtailment.
} 


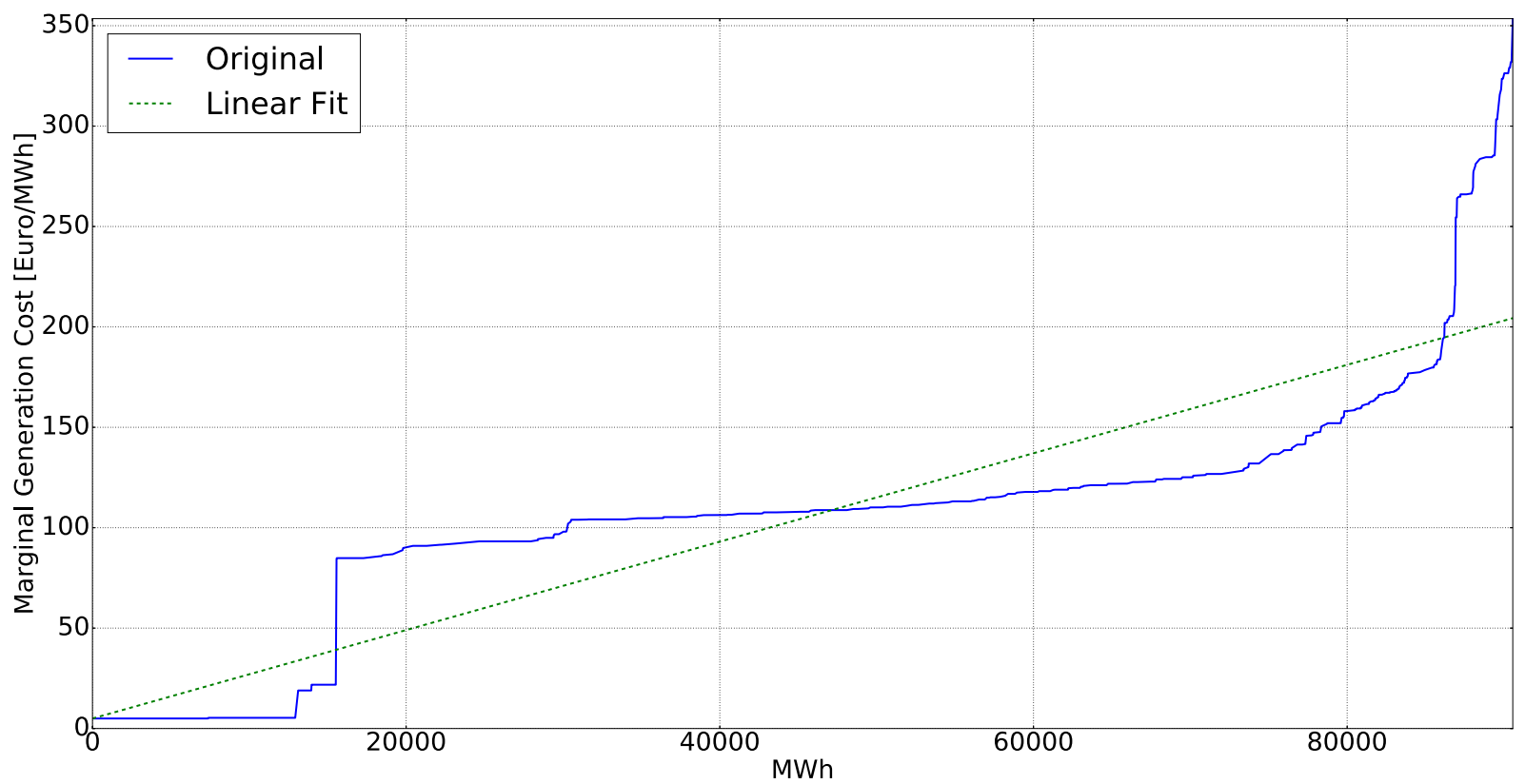

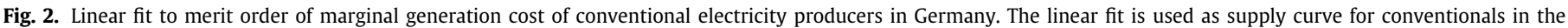
simulations of Section 4.

is moderate and there are no incentives in place from a RE support scheme, investment into renewables is such that there is very little costly curtailment.

For both policy scenarios with a RE support scheme, we choose a $70 \%$ target for the share of electricity generated from wind or solar. This constitutes an intermediate target given the range of 64\% to $97 \%$ as detailed in EU Energy Roadmap 2050 (cf. Section 1). ${ }^{11}$ We exogenously expand available storage capacity from zero to a value which is sufficiently high as to be quasi unlimited. Varying storage capacity enables us to obtain total system costs as a function of the storage level.

\subsection{Overview of main results}

Fig. 3 shows the total system cost $\mathscr{C}$ for different levels of the given storage capacity under the three scenarios. Three main insights emerge. First, it is evident that an increase in storage capacity strongly reduces system cost for low levels of installed storage capacity but marginal benefits (i.e., avoided cost) rapidly diminish as storage capacity increases. Marginal benefits from storage quickly approach zero at capacity levels of around $400 \mathrm{GWh}$, corresponding to 10.6 times the installed storage capacity in 2014 , or, equivalently, 6.5 average demand hours. This indicates that further discussion about the costs and benefits of storage capacity should concentrate on low to moderate levels of storage investment.

Second, the behavior of the storage operator for low to medium storage capacities (i.e., up to $400 \mathrm{GWh}$ ) shows exclusively intra-day storage cycles and no shifts of generation over seasons. We observe seasonal storage only for considerably higher installed storage capacities. Since the marginal benefits of storage capacity from avoided curtailment are zero for high values of installed capacity (i.e., beyond $400 \mathrm{GWh}$ ), it seems possible that the costs of seasonal storage exceed benefits, even in scenarios where the share of RE generation is as large as $70 \%$. This suggests that investments into short-term storage

$\overline{11}$ Note that unlike our RE target, these percentages also include electricity from hydro sources and biomass. technologies will possibly play a more important role as compared to longer-term, seasonal storage.

Third, the cost curve associated with a technology-specific RE support scheme shows that for low to medium storage capacities substantial savings in total system costs are possible. For low levels of storage capacity these can be as high as $11.4 \%$ of total system cost in a scenario without storage capacity and $7.7 \%$ if the current, installed storage capacity is assumed. This indicates that, given a fixed target for RE generation, improving the design of RE support schemes can either reduce total system cost in a scenario with given storage capacity or partially substitute for storage investment. ${ }^{12}$

The following subsections provide more detail about the market mechanisms behind these insights and provide further explanations and detailed results.

\subsection{The effects of adding storage capacity}

Storage capacity can act as a complement to intermittent renewables in that a storage operator has an incentive to fill the storage with cheap electricity in low-price hours, when there is abundant renewable generation, and to release electricity from storage in hours with high prices, when wind and solar generation is scarce. What is the value of adding storage capacity at the system or market-wide level?

\subsubsection{Total and marginal benefits of storage}

Fig. 3 provides measures for the total and marginal benefits of installing storage capacity: the total benefits of a given storage level are measured by the cost difference relative to a situation with zero storage; the marginal benefits are given by the negative derivative of the total system cost curve. With increasing storage capacity the total cost curves for the no-policy scenario as well as the two policy scenarios go towards a steady state which is reached when the constraints

\footnotetext{
12 Note that this result is independent from the actual need for RE support policies in the future. If in a situation where RE shares are high even without a subsidy policy integration cost can be lowered by altering the relative investment cost ratio of renewables the regulator can resort to a policy where the "subsidy" is zero for one technology and non-zero for the other.
} 


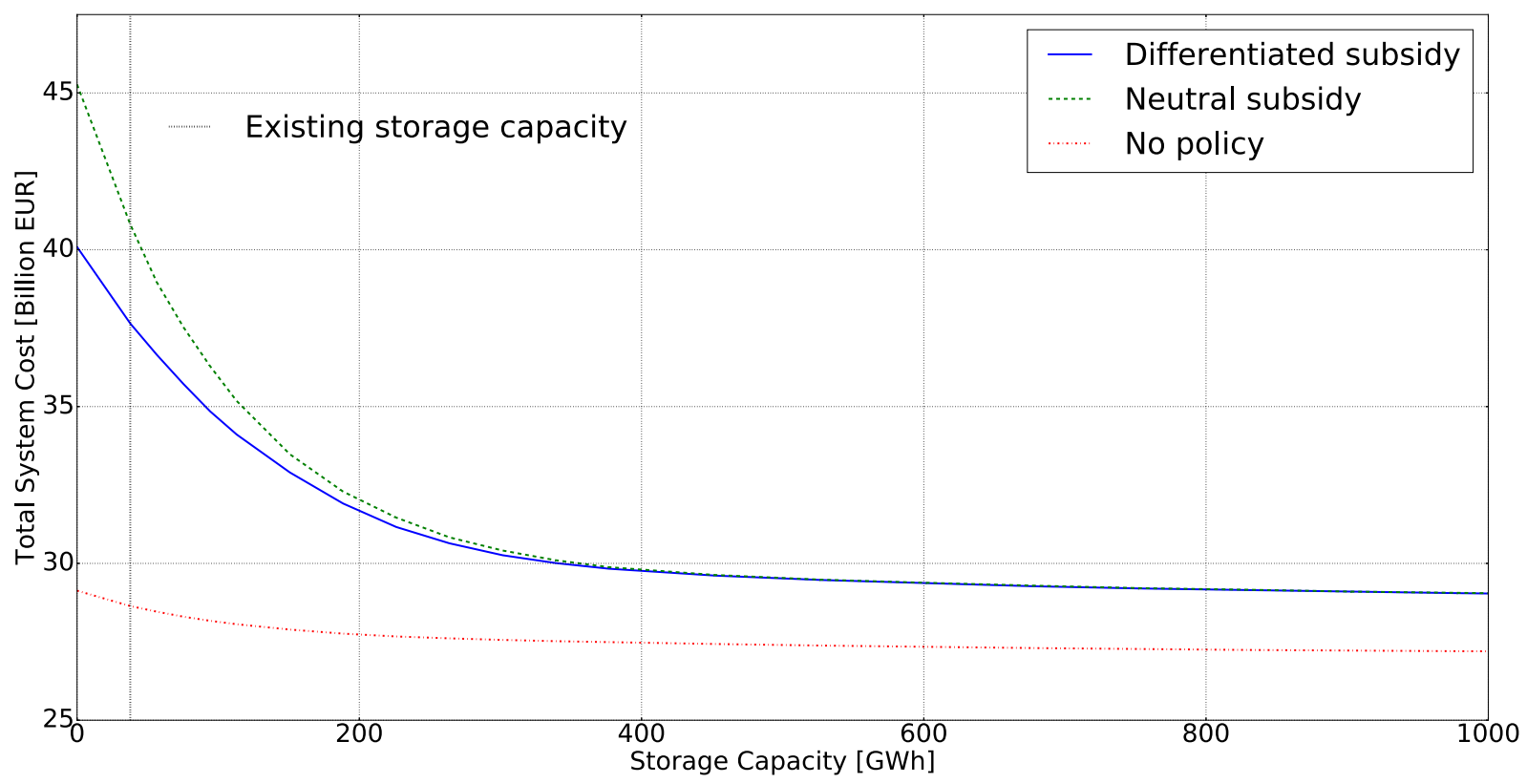

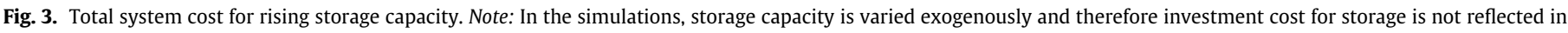
total system cost as defined in Eq. (20).

on storage are all non-binding and the intermittency of renewables is buffered by storage as much as possible. ${ }^{13}$ At this point, intermittency cost of RE is minimal and comparing total system cost at this point with total system cost for zero storage capacity allows us to gauge the maximum potential gains from storage (and, at the same time, total intermittency cost). Performing this calculation for the scenario with a Neutral subsidy to achieve a $70 \%$ RE target, we find that the maximum cost savings due to storage are $37 \%$ of total system cost with zero storage or $30 \%$ when using the currently installed storage capacity of our reference case. This number shows that from a system perspective, potential cost savings from storage are substantial but it also serves as an upper bound for the economically viable level of investment into storage.

For the actual storage investment decision of economic agents, marginal benefits of storage (alongside marginal cost) are crucial. Since the total cost curves go towards a steady state, their derivatives and thus marginal benefits go to zero. The decrease in marginal benefits of storage is steep so that from a system-wide perspective, most of the potential cost savings through storage capacity are achieved up to roughly $200 \mathrm{GWh}$. If we assume non-zero capital cost for storage, above this threshold, the incentives to add further storage capacity decrease rapidly even though cost savings in total system cost are still possible. We will further substantiate this argument in the cost-benefit analysis below.

We now take a closer look at what drives the benefits from additional storage. Storage reduces total system cost by preventing curtailment of generation from RE sources, thereby reducing the need for investment into RE capacity to meet a given RE target, i.e. investments into RE capacity are used more efficiently. Table 2 collects the relevant numerical results from our simulations. We report the values for key quantities such as total cost $\mathscr{C}$ and average investment cost $\kappa_{i}$ for both policy scenarios, Neutral subsidy and Differentiated subsidy, and storage capacity increasing from currently installed levels to unlimited storage. As shown in Fig. 3, there is a 30\% decrease in total cost with increasing storage for a Neutral subsidy from 40.8 Billion Euro to 28.5 Billion Euro. Together with total cost we report its two components, total investment cost per technology, $c_{i}^{\mathrm{i}}\left(I_{i}\right)$ for $i \in \mathscr{G}$, and conventional generation cost, $\sum_{t} c_{i t}^{\mathrm{g}}\left(X_{i t}\right)$ for $i \in \mathscr{B}$. With increasing storage, generation cost decreases due to storage substituting expensive conventional generation in peak hours, which results in overall lower fuel cost. As the numbers in Table 2 show, this is a reduction by $35 \%$ from current storage levels to unlimited storage, but the cost savings in absolute terms are small compared to cost savings in total renewable investment cost. The driver of cost reductions is total curtailment, $\sum_{t} C_{i t}$ for $i \in \mathscr{G}$, which decreases with increasing storage capacity and goes to zero. This is mirrored in the evolution of our efficiency measure $\kappa_{i}$ which reports average investment cost per net generation. For both RE technologies the average investment cost shows a decreasing trend with increasing storage consistent with the decrease in curtailment. ${ }^{14}$

Fig. 4 illustrates the impact of increasing storage capacity on RE investment by technology. We observe a steep decline in installed capacity for both technologies (associated with the increasing utilization efficiency) when storage is first introduced, which corresponds to a large marginal benefit of storage in this early stage of investments. As storage capacity increases until finally reaching a steady state, the relative share of solar power increases compared to wind as can also seen by the generation shares reported in Table 2 . This is the case because solar has cheaper investment cost per MW but is also inherently more volatile in its availability (having a daily period of zero output during nighttime and strong production peaks around noon). With rising storage capacity the disadvantages of this volatility disappear and make it more competitive relative to wind power.

\footnotetext{
13 Even if storage capacity is unconstrained energy loss over the storage cycle cannot be avoided because its roundtrip efficiency is below $100 \%$. This is a potential source for intermittency cost other than curtailment. The technology which is more likely to go into storage due to its availability pattern (in our case PV) then will contribute more to the remaining intermittency cost. For the impact of this effect on optimal subsidy differentiation see Sections 4.4 and 6 .
}

\footnotetext{
14 The slight increase in average investment cost for PV under unlimited storage stems from the fact that overall investment in solar is growing with increasing storage capacity and that rising marginal investment costs with degrading resource quality offset the gains from better utilization of capacity.
} 
Table 2

Overview of key impacts for alternative efficient RE support policies.

\begin{tabular}{|c|c|c|c|c|c|c|c|c|c|c|c|}
\hline \multirow[t]{2}{*}{ Storage factor ${ }^{\mathrm{a}}$} & \multicolumn{2}{|c|}{$\begin{array}{l}\text { Curtailment, } \\
\sum_{t} C_{i t}[\mathrm{TWh}]\end{array}$} & \multicolumn{2}{|c|}{$\begin{array}{l}\text { Gen. share by } \\
\text { tech. }(\%)^{\mathrm{b}}\end{array}$} & \multicolumn{2}{|c|}{$\begin{array}{l}\text { Av. investment cost, } \\
\kappa_{i}\left[\frac{E U R}{M W h}\right] \mathrm{c}\end{array}$} & \multicolumn{2}{|c|}{$\begin{array}{l}\text { Tot. inv. cost, } \\
c_{i}^{\mathrm{i}}\left(I_{i}\right)[\text { B. EUR] }\end{array}$} & \multirow{2}{*}{$\begin{array}{l}\text { Gen. cost, } \\
\sum_{i, t} c_{i t}^{\mathrm{g}}\left(X_{i t}\right) \\
\text { [B. EUR] }\end{array}$} & \multirow{2}{*}{$\begin{array}{l}\text { Tot. cost, } \\
\mathscr{C} \\
\text { [B. EUR] }\end{array}$} & \multirow{2}{*}{$\begin{array}{l}\text { Subsidy } \\
\text { diff. } \\
(\%)^{\mathrm{d}}\end{array}$} \\
\hline & Wind & PV & Wind & PV & Wind & PV & Wind & PV & & & \\
\hline \multicolumn{12}{|l|}{ Neutral subsidy } \\
\hline 1 (37.7 GWh) & 46.3 & 94.0 & 30.5 & 39.5 & 94.2 & 84.9 & 15.5 & 18.1 & 7.1 & 40.8 & 100 \\
\hline $5(188.5 \mathrm{GWh})$ & 12.5 & 21.3 & 27.4 & 42.6 & 74.8 & 62.2 & 11.3 & 14.7 & 6.3 & 32.3 & 100 \\
\hline 10 (377 GWh) & 1.8 & 3.6 & 24.6 & 45.4 & 67.7 & 57.9 & 9.4 & 14.8 & 5.7 & 29.9 & 100 \\
\hline Unlim. & 0 & 0 & 20.1 & 49.9 & 64.4 & 58.1 & 7.4 & 16.5 & 4.6 & 28.5 & 100 \\
\hline \multicolumn{12}{|c|}{ Differentiated subsidy } \\
\hline 1 (37.7 GWh) & 44.7 & 29.2 & 39.8 & 30.2 & 94.4 & 64.3 & 20.3 & 10.5 & 6.8 & 37.6 & 62 \\
\hline $5(188.5 \mathrm{GWh})$ & 12.0 & 11.5 & 31.3 & 38.7 & 76.0 & 58.8 & 13.2 & 12.6 & 6.2 & 31.9 & 74 \\
\hline 10 (377 GWh) & 1.8 & 2.3 & 26.4 & 43.6 & 68.6 & 57.2 & 10.2 & 13.4 & 5.7 & 29.8 & 84 \\
\hline Unlim. & 0 & 0 & 21.7 & 48.3 & 65.2 & 57.7 & 8.0 & 15.9 & 4.6 & 28.5 & 91 \\
\hline
\end{tabular}

a Storage factor denotes storage capacity in multiples of the currently installed capacity $(37.7 \mathrm{GWh})$.

b Note that the generation shares always add up to the policy target of $70 \%$.

c Average investment cost is measured in annuitized investment cost per generation net of curtailment.

${ }^{d}$ We report the percentage value of the subsidy per MWh for PV relative to wind.

\subsubsection{Volatility of equilibrium electricity prices}

The diminishing volatility for an increasing storage capacity is also reflected by a reduced dispersion of equilibrium electricity prices on hourly wholesale markets. A comparison across the Panels (a)-(d) in Fig. 5 shows that the price volatility sharply reduces as storage capacity increases, reaching its theoretical minimum when storage capacity is unlimited. ${ }^{15}$

\subsubsection{Intra-day vs. seasonal storage}

The maximally observed reduction in price volatility is only possible when storage capacity is very large or unlimited and the storage operator engages in shifting electricity generation over seasons in addition to shorter storage cycles. To illustrate the two principal ways how storage operates in the electricity market over the different time scales, Fig. 6 contrasts the behavior of a profit-maximizing storage operator for a situation with constrained (equal to $377 \mathrm{GWh}$ ) and unlimited (equal to $6032 \mathrm{GWh}$ ) storage capacity. The following insights emerge.

First, there is a short-term consideration associated with the intra-day storage of electricity which aims at exploiting the price differentials between low-price and high-price periods over a typical day. Optimization over this short-term cycle is closely associated with solar generation and shifts excess PV generation from daytime hours to hours with little or no solar availability. Intra-day storage optimization is present for cases with both constrained and unlimited storage capacity. Second, when constraints on storage are lifted, we observe a long-term behavior with seasonal storage where reserves are filled over the summer months (mostly with solar generation) and depleted in winter and spring periods when solar energy is scarce. When the storage level is 10 times the base-year level $(377 \mathrm{GWh})$, the marginal cost savings have become small and are rapidly decreasing towards zero. This suggests that, even with a relatively aggressive RE target of $70 \%$, seasonal storage is not feasible as the necessary capacity investments will neither pay off for investors nor do they substantially reduce the total system cost (or increase the market surplus) to society.

\subsection{Differentiated renewable energy support schemes}

Mandating that a large share of electricity has to come from intermittent RE has been shown to cause substantial system integration costs (see Section 4.3 and the text around Fig. 3). One strategy for buffering volatility and to reduce system integration cost (i.e., curtailment) is to increase storage capacity. Coping with volatility through this channel, however, is subject to a trade-off between the cost savings in system integration cost and the rising cost for storage investment-and we have shown that the marginal benefits from additional storage investments rapidly diminish at the system level. An alternative buffering mechanism is through optimizing the regulatory design of the RE support scheme in order to take advantage of the complementarity of the underlying natural resources and their correlation with time-varying electricity demand.

Our key finding here is that a technology-neutral RE support scheme (for example, implemented as a per MWh subsidy on RE output) is not a cost-effective strategy to reach a given RE target at lowest cost to society. The cost of achieving a given RE target can be significantly lowered by optimally differentiating the policy support among RE technologies. ${ }^{16}$

As there are interdependencies between both buffering strategies (i.e., differentiated RE subsidies and enhanced storage capacity), we analyze the potential of differentiated RE support for different levels of storage capacity. Table 2 reports the optimal differentiation of the subsidy for solar compared to wind. For the current storage capacity, solar receives only $62 \%$ of wind subsidy per MWh produced. Solar energy has lower investment cost per MW and its resource availability is highly concentrated during a few hours of the day. With a high targeted share of RE and with low storage capacity, this implies a much higher curtailment of solar energy as compared to wind. The lower subsidy leads to a shift of investment from PV to wind, thereby lowering the average investment cost for solar as the remaining solar capacity can be used more efficiently. Accordingly, average investment cost $\kappa_{i}$ for solar for each storage level is lower than the corresponding value with a neutral subsidy. As a direct consequence, with an optimally differentiated subsidy we observe lower total system cost

\footnotetext{
15 The remaining price variation under unlimited storage capacity is due to roundtrip efficiency losses. As we assume a $25 \%$ loss of energy over the storage cycle, there needs to be a price spread between periods of injection into and release from storage for the storage activity to be economically viable.
}

16 Note, however, that technology-differentiation is not the only possible way to address these issues. Other subsidy designs such as a per MW subsidy or a subsidy per MWh which is only given for generation which is not curtailed could also be considered. 


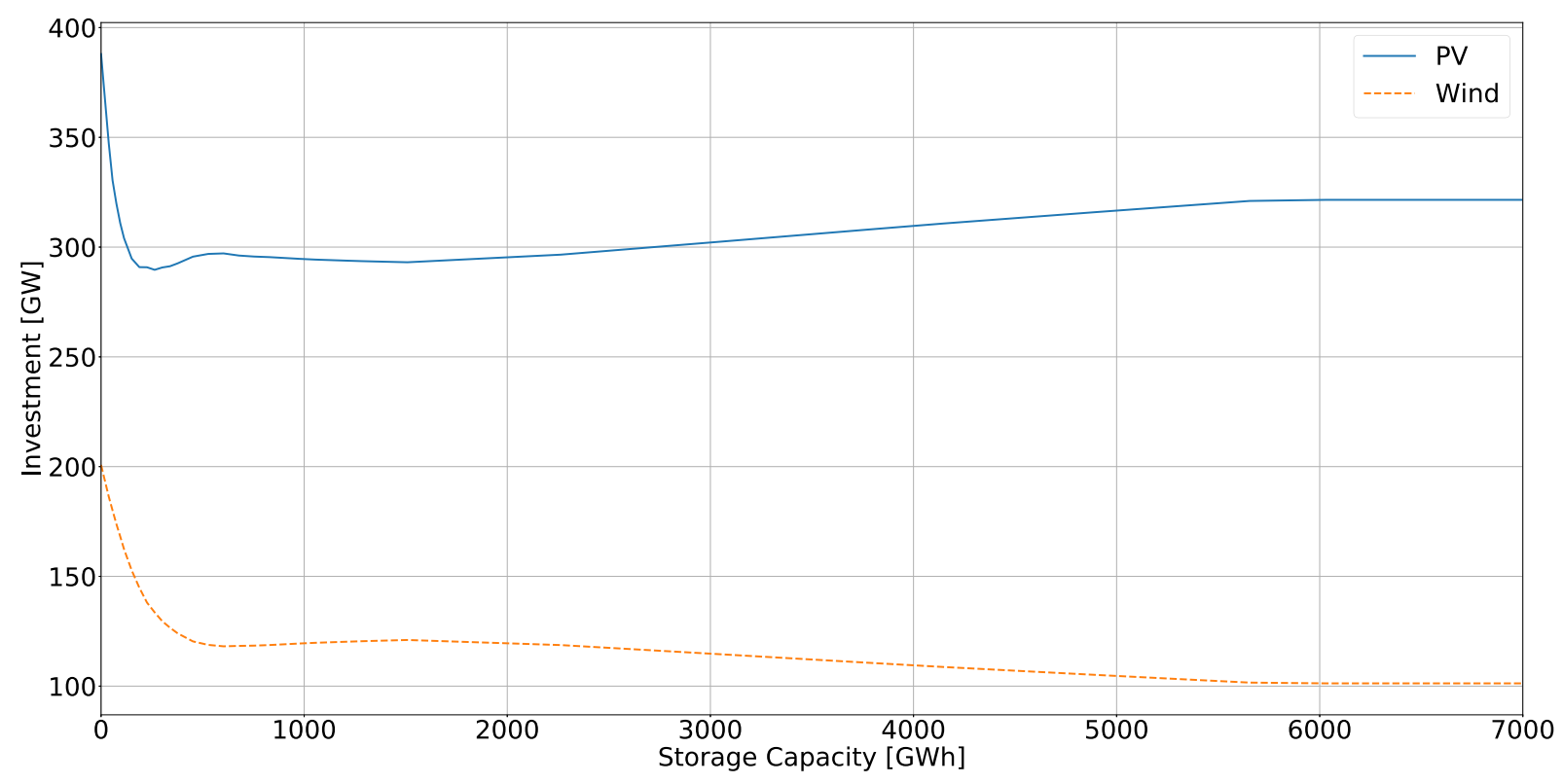

Fig. 4. RE investment with increasing storage capacity.

for low to medium storage capacities where curtailment of RE generation is necessary. With increasing storage, curtailment is reduced to zero and the motive for differentiation vanishes almost completely and remains at $91 \%$. This moderate differentiation is explained by storage efficiency losses because generation from solar is more likely to go into storage than generation from wind.

Similar to increased storage capacity, differentiated RE support brings about a reduction in curtailment-however, the mechanism is different. Under a neutral RE subsidy, investments into RE technologies are chosen such that marginal investment costs are equal across the two technologies. Due to the intermittent nature of RE sources this causes demand and supply to be mismatched in a large number of periods with the implication of high (and costly) curtailment. Since the subsidy is designed in a way that generators receive additional revenue for each unit produced, even though this unit may have to be curtailed, agents do not take into account the mismatch of demand and supply, i.e. they do not properly internalize the curtailment costs associated with more volatile RE supply when taking their

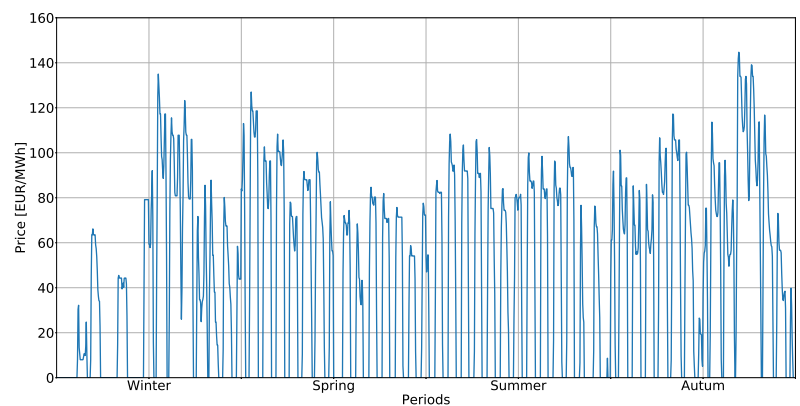

(a) Storage level 1 (37.7 GWh).

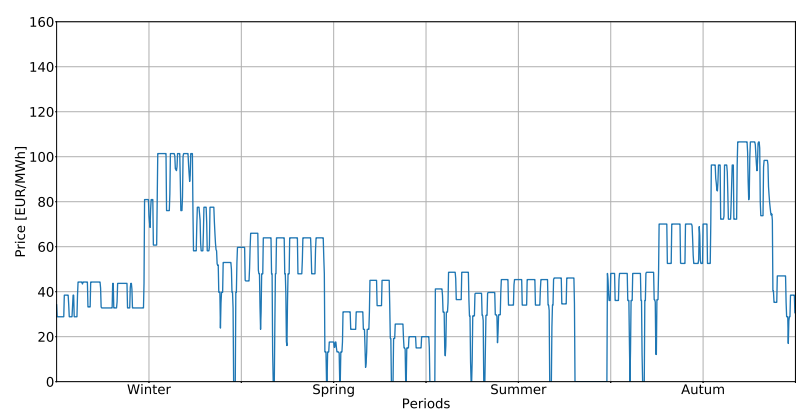

(c) Storage level 10 (377 GWh).

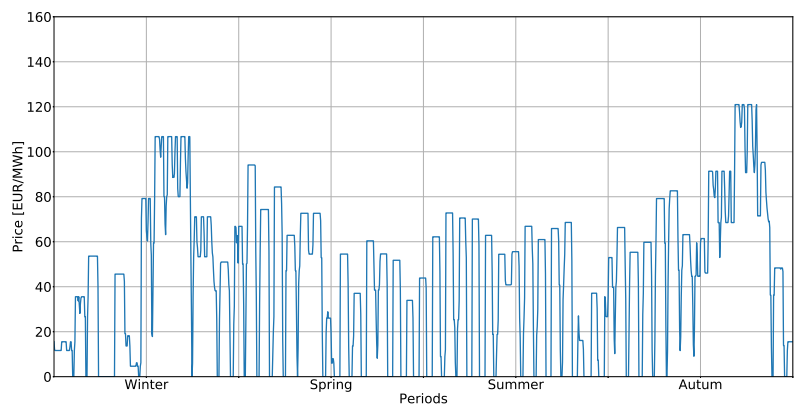

(b) Storage level 5 (188.5 GWh).

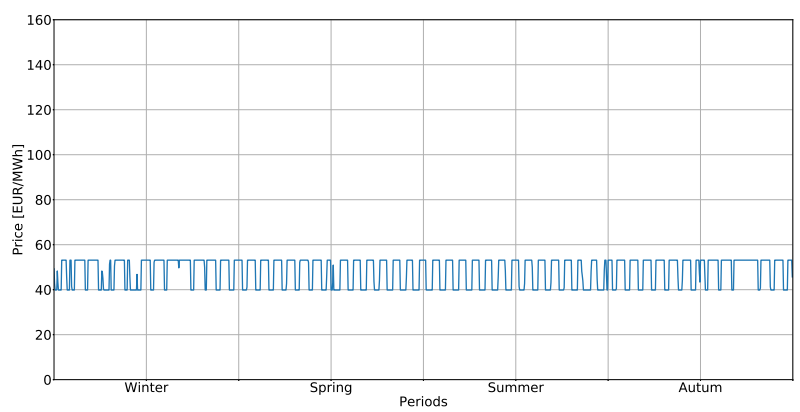

(d) Unlimited storage.

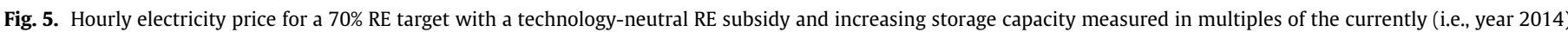
installed level. 


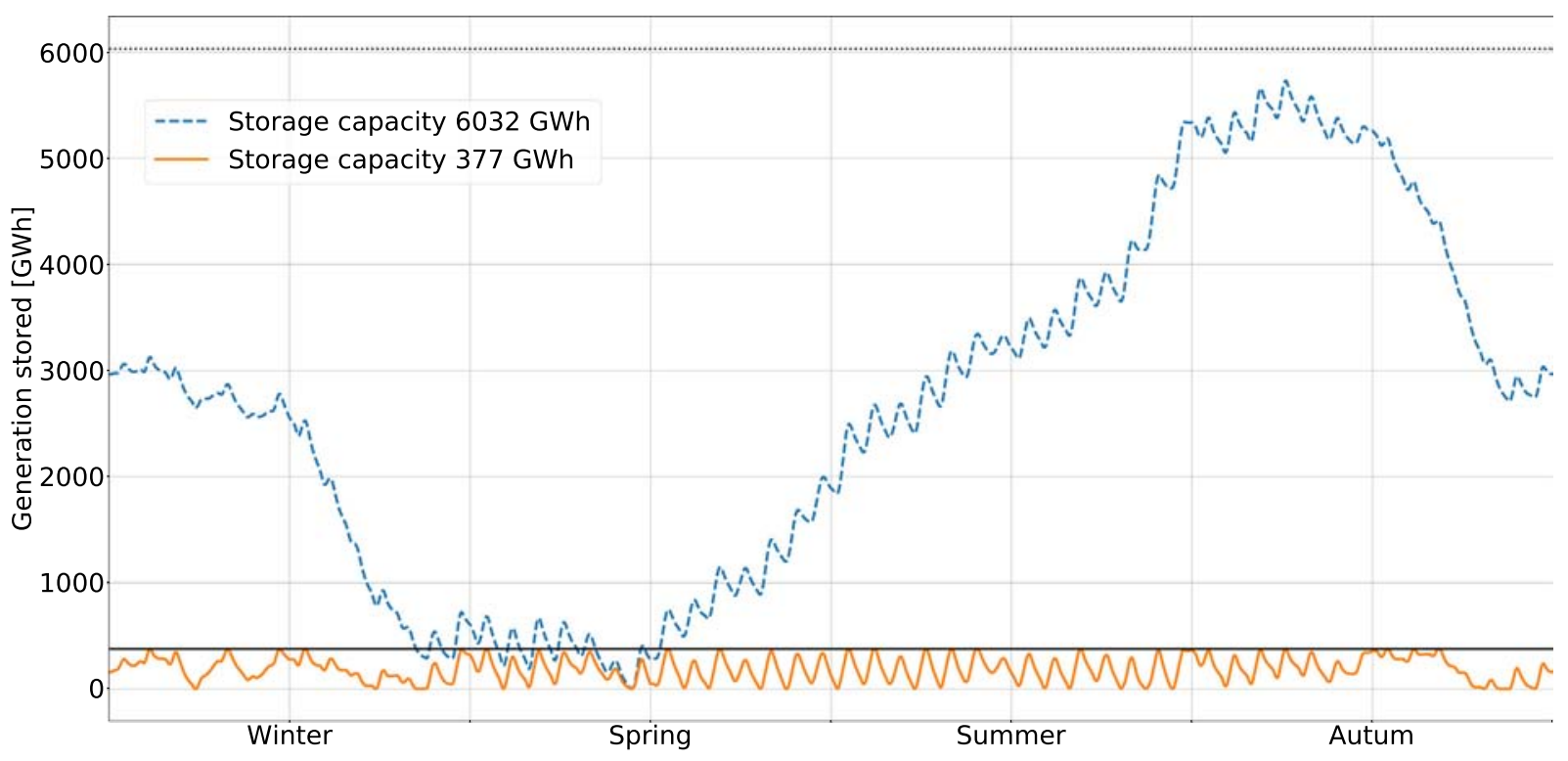

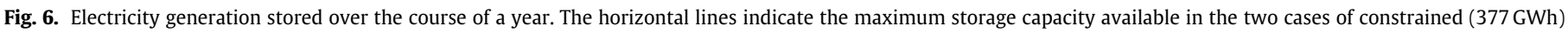
and unlimited storage capacity. Under unlimited storage, the capacity of $6032 \mathrm{GWh}$ is never exhausted.

investment decisions. In contrast, optimal differentiation of the RE subsidy induces investment patterns such that the marginal investment costs can differ between the two intermittent RE technologies. Total system costs are reduced as curtailment decreases due to a "better usability" of electricity, i.e. by exploiting the complementarities with respect to the availability of the underlying natural resource and its correlation with electricity demand. To a smaller extent, when storage efficiency is below $100 \%$, differentiation favors the technology which is less dependent on storage, reducing energy losses and thus total system cost are also reduced.

\section{How much energy storage?-A simple cost-benefit analysis}

This section explores the question how much energy storage is optimally needed to achieve a certain share of intermittent RE at the lowest cost to society. In trying to tackle this question, we keep the conceptual framework deliberately simple and adopt a canonical cost-benefit analysis based on the equalization of marginal benefits and marginal costs. The main idea is as follows. First, we make use of the detailed electricity simulation model presented in Section 4 to characterize the marginal benefits of energy storage. Second, we obtain estimates for the marginal costs of energy storage by briefly reviewing the relevant literature. Third, the optimal level of energy storage capacity is then determined based on a comparison of marginal costs and benefits. We also examine how the choice of regulatory design-with respect to a technology-neutral or technology-specific support mechanism which has been shown to act as a potential buffer against the market volatility induced by intermittent RE technologies-affects the optimal level of storage.

\subsection{Marginal costs curve}

For the construction of the marginal costs of installing different levels of energy storage, we refer to empirical estimates documented in the literature. Characterizing marginal costs over a large range of storage levels is, of course, fraught with large difficulties. First, the investment costs for both current and future storage technologies are highly uncertain. Second, reliable estimates for the potential of different storage technologies are also subject to considerable uncertainty.

We rely on cost estimates for storage technologies from Zakeri and Syri (2015) and on estimates for the potential of different technologies from Hartmann et al. (2012). ${ }^{17}$ Table 3 summarizes these estimates which provide the basis for deriving a marginal cost curve for storage capacity. We construct the marginal cost curve as a step function with horizontal steps corresponding to the installation cost of the respective storage technology and with the length of the horizontal lines corresponding to the respective potential. Fig. 7 shows the cost curves for the Low and Medium cost assumptions. Note that we show only the lowest step which represents the cheapest optioncompressed air energy storage. The other parts of the step-function to the right of the lowest step are not relevant for our discussion given the range of storage level spanned by the marginal benefits curves.

\subsection{Marginal benefits curve}

We construct the marginal benefits curve for energy storage based on the simulations of the wholesale electricity market model described in Section 4. Specifically, we use model estimates of how total system costs change with different levels of energy storage (see Fig. 3). The marginal benefit of adding a small amount of storage capacity is equal to the negative derivative of the total cost curve. Numerically, this is approximated by the difference quotient of total cost with respect to storage capacity evaluated for different storage levels. Let $\mathscr{C}^{*}\left(\bar{k}^{\Sigma}\right)$ denote the equilibrium cost for a given storage level $\bar{k}^{\Sigma}$. The marginal benefits of energy storage, $\beta\left(\bar{k}^{\Sigma}\right)$, are then given by:

$\beta\left(\bar{k}^{\Sigma}\right)=-\frac{\mathscr{C}^{*}\left(\bar{k}^{\Sigma}\right)-\mathscr{C}^{*}\left(\bar{k}^{\Sigma}-h\right)}{h}$,

17 Comparing to other studies, cost estimates by Zakeri and Syri (2015) are at the lower end of the cost range. Using rather low cost estimates, our results are conservative in the sense of being favorable for storage investments. However, the available studies cannot incorporate highly uncertain future cost reductions due to technological breakthroughs. Thus, the given cost estimates represent a current state for these technologies. 
Table 3

Estimates taken from the literature to construct the marginal cost curve for storage.

\begin{tabular}{lllll}
\hline & \multicolumn{2}{l}{ Cost [million EUR/GWh] } & \multirow{2}{*}{ Potential [GWh] } \\
\cline { 2 - 4 } Technology & Low & Medium & High & \\
\hline Pumped hydro storage & 96 & 137 & 181 & 2000 \\
Compressed air energy storage & 48 & 92 & 106 & 27000 \\
Power to gas & 227 & 262 & 457 & 380000 \\
Batteries $^{\mathrm{a}}$ & 368 & 427 & 659 & -b $^{\mathrm{b}}$ \\
\hline
\end{tabular}

Notes: Cost estimates are taken from Zakeri and Syri (2015) and estimates of potentials from Hartmann et al. (2012).

a The values for batteries are own calculations of average values over different battery technologies in Zakeri and Syri (2015).

b Since batteries are not subject to similar physical restrictions as mechanical storage technologies, their maximum potential is likely to be very high. We refrain from reporting a value on this since it is highly uncertain.

where $h$ denotes the step size, i.e. the difference between single points on the storage capacity axis. ${ }^{18}$ Fig. 7 depicts the numerical marginal benefits function for each of the two RE policy cases (i.e., Neutral subsidy and Differentiated subsidy).

\subsection{Optimal storage capacity and the impact of technology-specific RE policy}

The simple cost-benefit framework depicted by Fig. 7 enables us to draw several conclusions. First, given the available cost estimates for storage, the economically optimal storage capacity to integrate intermittent RE supply consistent with a 70\% RE target is moderate in any case: under Medium cost assumptions, and a technology-neutral RE support, roughly doubling the level of existing capacities would be sufficient for the German electricity market; under Low cost assumptions, the optimal storage level is about $150 \mathrm{GWh}$ or four times larger than the currently installed level. These findings are in line with large parts of the literature; see, for example, Zerrahn et al. (2018) and the studies cited therein. Zerrahn et al. (2018) find that for a RE target of $70 \%$, the optimal storage level is $230 \mathrm{GWh}$ which lies both within the range of estimates obtained by our approach as well as the bulk of the literature.

Second, Fig. 7 visualizes the striking impact of carefully designed technology-specific RE support on the optimal level of energy storage. Optimal differentiation of RE subsidies reduces curtailment and thus the marginal benefits from storage. As a result, the optimal level of storage capacity is considerably lower: under Medium cost assumptions, no additional investment into storage beyond current installations is needed; under Low storage cost, a storage capacity of approximately $90 \mathrm{GWh}$ is optimal-almost half of what would be optimally needed under a technology-neutral RE support. This suggests that coping with the volatility induced by intermittent RE sources can be achieved to a large extent through smart policy design which subsidizes RE technologies according to their heterogeneous value for system integration cost (rather than determining the level of subsidies based on a narrow consideration of investment costs per MW of production capacity).

\section{Sensitivity analysis}

We check the robustness of our main findings by varying parameter values along two important dimensions, the policy target for the RE share in production (denoted by $\gamma$ in Section 2) and the roundtrip efficiency of storage (denoted by $\psi$ in Section 2). Table 4 reports values for several key variables of our analysis for the parameter choice in our main results section $(\gamma=70 \%, \psi=75 \%)$ which we

\footnotetext{
18 For practical reasons, we choose $h$ to coincide with the numerical step size that was used to obtain the simulations in Section 4.
}

denote as core case and for several alternative scenarios: high efficiency $(\gamma=70 \%, \psi=90 \%)$, low efficiency $(\gamma=70 \%, \psi=60 \%)$, and high RES ( $\gamma=80 \%, \psi=75 \%)$.

\subsection{Higher required RE share}

With rising shares of RE generation the impact of the intermittency of wind and solar resources on the total electricity system becomes more pronounced and the mismatch between demand and supply more severe. We explore the implications of a $10 \%$ increase in RE generation from $70 \%$ to $80 \%$ of total generation and report the results in Table 4 in columns four and eight (H. RES) for a neutral subsidy and a differentiated subsidy, respectively. Due to the higher RE share requirements total cost and curtailment increase considerably when the available storage capacity is low. We find, however, very similar characteristics as in the core case. As expected, rising storage capacity decreases cost of curtailment (as can be seen from the decrease in total cost when storage capacity increases). In line with our findings for the core case, differentiation of the subsidy has the potential to reduce total cost considerably for low levels of storage capacity. At current levels of storage (storage factor 1), subsidy differentiation reduces total system cost by $30 \%$, which is a larger effect than was observed in the core case. Because more generation from renewables needs to be integrated in the system our cost-benefit analysis yields also considerably higher optimal storage capacity (309 GWh, roughly eight times the current capacity) which can be reduced by $29 \%$ through subsidy differentiation. Fig. 8 is an updated version of Fig. 6 for the high RES scenario and we observe that at a storage capacity of $377 \mathrm{GWh}$ (ten times the currently installed capacity) and well above the optimal capacity with a neutral subsidy scheme ( 309 GWh) we do not observe a clear seasonal storage behavior as the one we observe for unlimited storage capacity $(6786 \mathrm{GWh})$ in the same figure.

\subsection{Efficiency of storage}

The efficiency of storage (capturing the energy losses over one full storage cycle of injection, storage, and release of electricity) varies considerably over different storage technologies and is a major determinant of their overall performance and impact on the electricity system. The efficiency $\psi=75 \%$ which is used for the core case simulations is in line with values reported for hydro storage (see, e.g. Egerer et al., 2014; Newbery, 2016; Zakeri and Syri, 2015). For other promising technologies such as compressed air energy storage and batteries, efficiency varies within a wide of about $60 \%$ to $90 \%$ (see Table B1 in Zakeri and Syri, 2015). We therefore add two scenarios with these extreme values for efficiency, high efficiency and low efficiency, to our sensitivity analysis capturing different technology choices for storage. The results are reported in Table 4 in columns two and three for a neutral subsidy and columns six and seven for a differentiated subsidy.

A comparison of the results for high and low storage efficiency with the core case shows relatively small changes for most variables. The overall effect on total cost compared to the core case is small for limited current storage capacity (e.g., with neutral subsidy and high efficiency total cost increases by $0.2 \%$ and with low efficiency it decreases by $0.2 \%$ ) and for the extreme case of unlimited storage total cost decreases by $4.9 \%$ and increases by $5.6 \%$ for high efficiency and low efficiency, respectively. Not surprisingly, when efficiency is higher (lower), the optimal storage capacity is also higher (lower), both for the case of a neutral subsidy and a differentiated subsidy.

In both scenarios, subsidy differentiation reduces total cost by a similar amount than in the core case and the optimal storage capacity can be significantly reduced compared to a neutral subsidy scheme. It is interesting to note, that the optimal differentiation depends on the storage efficiency. In the core case, we still observe an optimal differentiation that reduces the subsidy from solar even if storage 


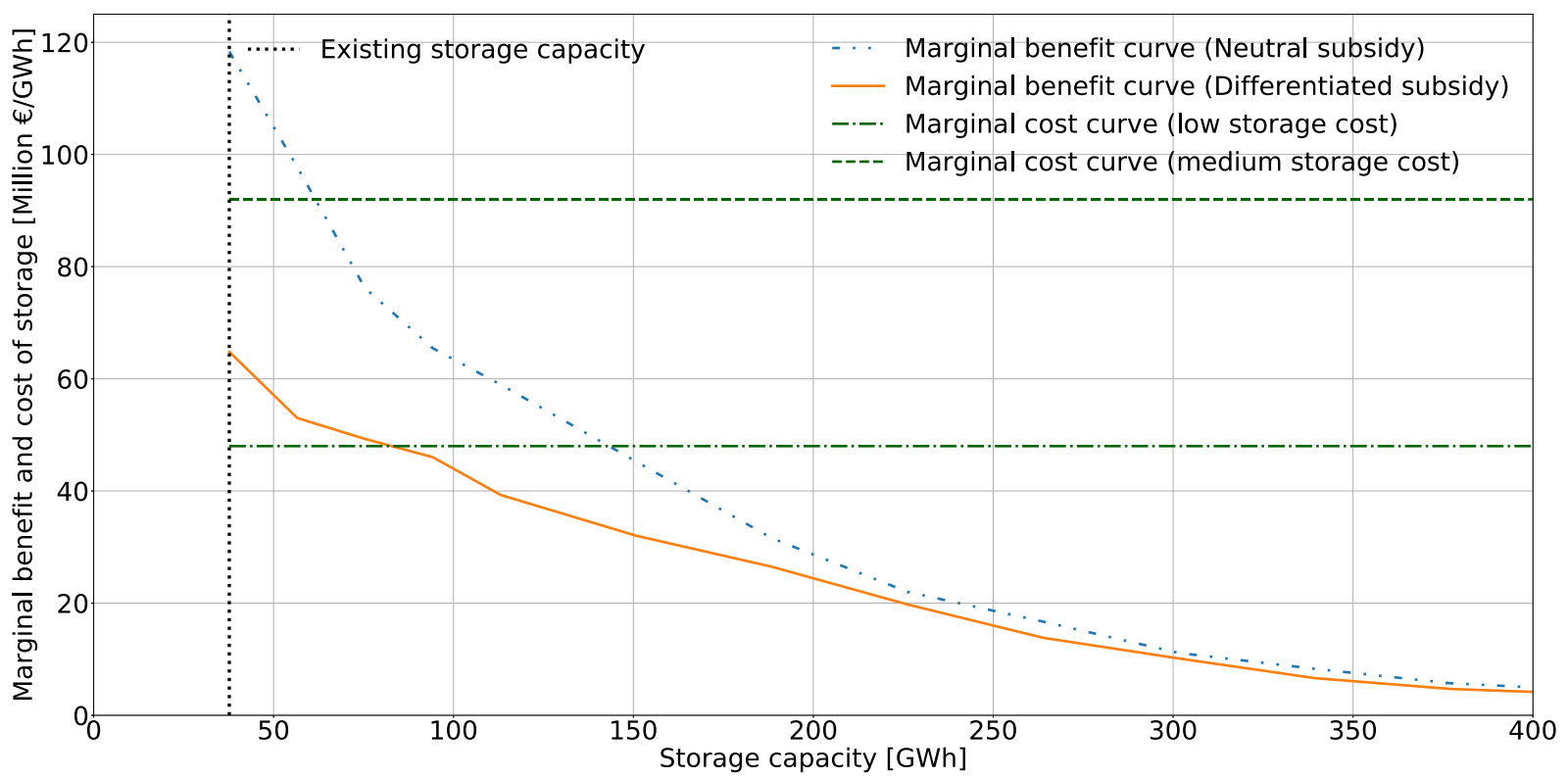

Fig. 7. Marginal costs and benefits for different levels of energy storage and technology-neutral and technology-specific RE support schemes.

capacity is unlimited and curtailment is zero. This is because electricity from solar energy is more likely to go into storage compared to wind due to its highly concentrated availability pattern. If storage losses are nonzero this makes solar less valuable than wind and a small differentiation of the subsidy persists in the optimum. Accordingly, we observe that with a higher storage efficiency the optimal

Table 4

Sensitivity analysis.

\begin{tabular}{|c|c|c|c|c|c|c|c|c|c|c|}
\hline \multirow[b]{2}{*}{ Variable } & \multirow[b]{2}{*}{ Tech. } & \multirow[b]{2}{*}{ Storage factor ${ }^{\mathrm{a}}$} & \multicolumn{4}{|c|}{ Neutral } & \multicolumn{4}{|c|}{ Differentiated } \\
\hline & & & Core & H. Eff. & L. Eff. & H. RES & Core & H. Eff. & L. Eff. & H. RES \\
\hline \multirow[t]{4}{*}{ Total cost [B. EUR] } & & 1 (37.7 GWh) & 40.8 & 40.9 & 40.7 & 73.8 & 37.6 & 37.6 & 37.8 & 51.4 \\
\hline & & $5(188.5 \mathrm{GWh})$ & 32.3 & 32.0 & 33.2 & 44.2 & 31.9 & 31.4 & 32.8 & 39.5 \\
\hline & & $10(377 \mathrm{GWh})$ & 29.9 & 28.8 & 31.1 & 34.3 & 29.8 & 28.8 & 31.0 & 33.7 \\
\hline & & Unlim. & 28.5 & 27.1 & 30.1 & 30.8 & 28.5 & 27.1 & 30.1 & 30.8 \\
\hline \multirow[t]{8}{*}{ Curtailment [TWh] } & Wind & 1 (37.7 GWh) & 46.3 & 58.9 & 44.7 & 115 & 44.7 & 43.5 & 43.7 & 104 \\
\hline & & $5(188.5 \mathrm{GWh})$ & 12.5 & 19.9 & 12.9 & 45 & 12.0 & 13.1 & 11.7 & 46 \\
\hline & & $10(377 \mathrm{GWh})$ & 1.8 & 1.9 & 2.2 & 4.3 & 1.8 & 2.0 & 1.5 & 8.3 \\
\hline & & Unlim. & 0 & 0 & 0 & 0 & 0 & 0 & 0 & 0 \\
\hline & PV & 1 (37.7 GWh) & 94.0 & 84.9 & 90.6 & 380 & 29.2 & 32.0 & 32.3 & 79 \\
\hline & & $5(188.5 \mathrm{GWh})$ & 21.3 & 18.5 & 21.8 & 118 & 11.5 & 11.6 & 12.3 & 39 \\
\hline & & $10(377 \mathrm{GWh})$ & 3.6 & 4.2 & 3.0 & 31 & 2.3 & 2.9 & 1.7 & 15 \\
\hline & & Unlim. & 0 & 0 & 0 & 0 & 0 & 0 & 0 & 0 \\
\hline \multirow{8}{*}{$\begin{array}{l}\text { Generation share by technology } \\
(\%)^{\mathrm{b}}\end{array}$} & Wind & 1 (37.7 GWh) & 30.5 & 28.4 & 30.6 & 33.9 & 39.8 & 40.4 & 38.9 & 50.6 \\
\hline & & 5 (188.5 GWh) & 27.4 & 26.5 & 27.6 & 29.5 & 31.3 & 31.9 & 31.4 & 38.8 \\
\hline & & $10(377 \mathrm{GWh})$ & 24.6 & 24.3 & 25.5 & 27.6 & 26.4 & 25.8 & 27.9 & 31.7 \\
\hline & & Unlim. & 20.1 & 17.6 & 22.5 & 21.9 & 21.7 & 18.3 & 25.0 & 24.7 \\
\hline & PV & 1 (37.7 GWh) & 39.5 & 41.6 & 39.4 & 46.1 & 30.2 & 29.6 & 31.1 & 29.4 \\
\hline & & $5(188.5$ GWh $)$ & 42.6 & 43.5 & 42.4 & 50.5 & 38.7 & 38.1 & 38.6 & 41.2 \\
\hline & & $10(377 \mathrm{GWh})$ & 45.4 & 45.7 & 44.5 & 52.4 & 43.6 & 44.2 & 42.1 & 48.3 \\
\hline & & Unlim. & 49.9 & 52.4 & 47.5 & 58.1 & 48.3 & 51.7 & 45.0 & 55.3 \\
\hline \multirow{8}{*}{ Average investment cost $\left[\frac{E U R}{M W h}\right]^{c}$} & Wind & 1 (37.7 GWh) & 94.2 & 102 & 93.3 & 133 & 94.4 & 93.9 & 93.8 & 123 \\
\hline & & $5(188.5 \mathrm{GWh})$ & 74.8 & 78.6 & 75.3 & 93.3 & 76.0 & 76.6 & 76.2 & 95 \\
\hline & & $10(377 \mathrm{GWh})$ & 67.7 & 67.2 & 68.8 & 70.7 & 68.6 & 68.0 & 69.6 & 74.6 \\
\hline & & Unlim. & 64.4 & 62.7 & 66.1 & 65.6 & 65.2 & 63.0 & 67.5 & 67.1 \\
\hline & PV & 1 (37.7 GWh) & 84.9 & 81.4 & 83.7 & 180 & 64.3 & 65.4 & 65.4 & 84.3 \\
\hline & & $5(188.5 \mathrm{GWh})$ & 62.2 & 61.4 & 62.4 & 88.6 & 58.8 & 58.6 & 59.2 & 67.1 \\
\hline & & $10(377 \mathrm{GWh})$ & 57.9 & 57.8 & 57.8 & 66.0 & 57.2 & 57.2 & 56.9 & 61.4 \\
\hline & & Unlim. & 58.1 & 58.2 & 57.9 & 60.1 & 57.7 & 58.1 & 57.3 & 59.4 \\
\hline \multirow[t]{4}{*}{ Subsidy diff. (\%) ${ }^{\mathrm{d}}$} & & 1 (37.7 GWh) & 100 & 100 & 100 & 100 & 62 & 62 & 65 & 53 \\
\hline & & $5(188.5 \mathrm{GWh})$ & 100 & 100 & 100 & 100 & 74 & 72 & 76 & 67 \\
\hline & & $10(377 \mathrm{GWh})$ & 100 & 100 & 100 & 100 & 84 & 85 & 81 & 78 \\
\hline & & Unlim. & 100 & 100 & 100 & 100 & 91 & 96 & 86 & 90 \\
\hline Optimal storage capacity [GWh] & & & 143 & 150 & 123 & 309 & 83 & 98 & 72 & 219 \\
\hline
\end{tabular}

a Storage factor denotes storage capacity in multiples of the currently installed capacity ( $37.7 \mathrm{GWh})$

b Note that the generation shares always add up to the policy target of $70 \%$.

c Average investment cost is measured in annuitized investment cost per generation net of curtailment.

d We report the percentage value of the subsidy per MWh for PV relative to wind. 


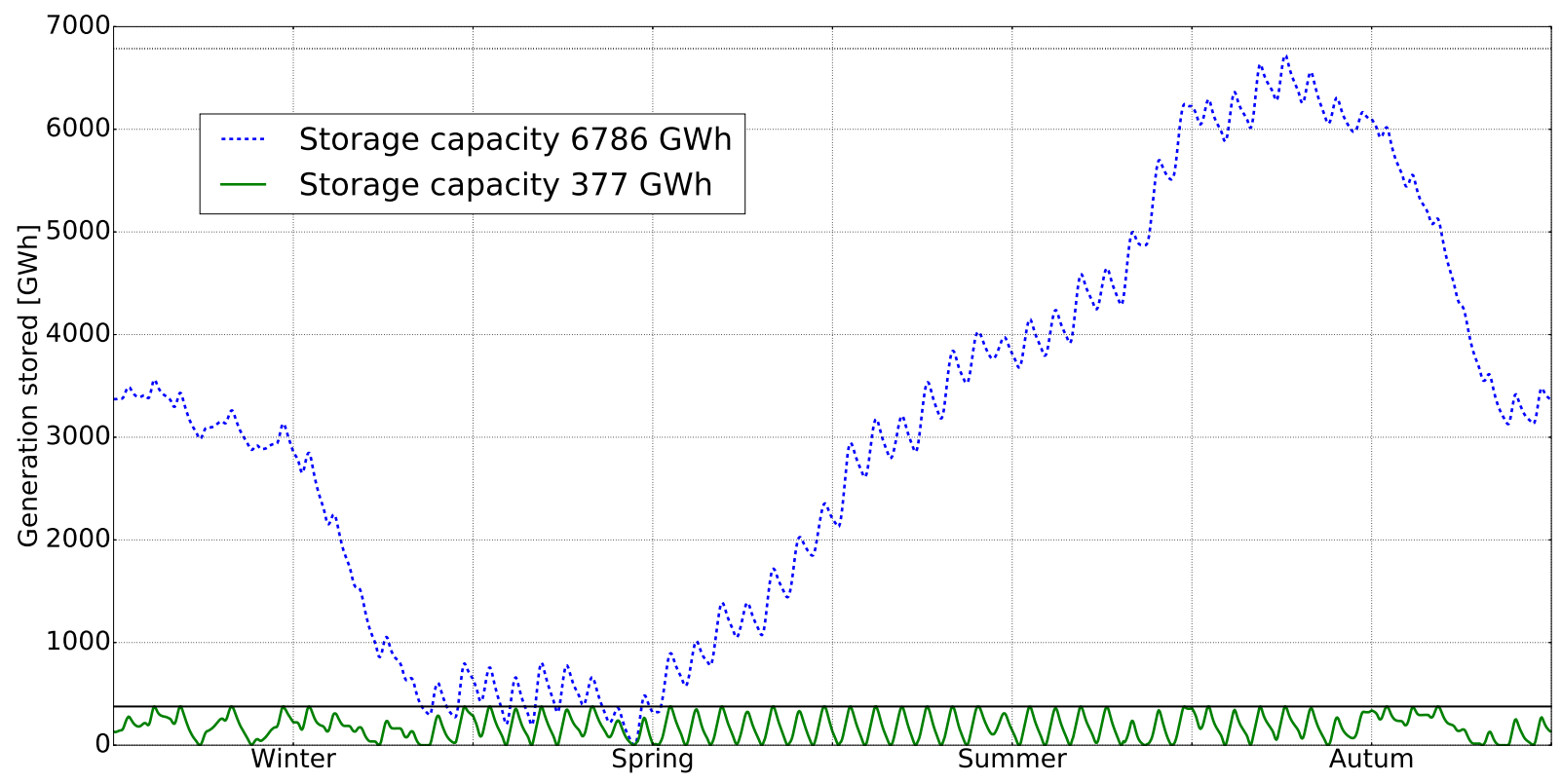

Fig. 8. Seasonal storage patterns for quasi unlimited storage capacity ( $6786 \mathrm{GWh})$ and ten times the current storage capacity ( $377 \mathrm{GWh})$.

differentiation for unlimited storage capacity is closer to a neutral scheme and vice versa for lower efficiency. The numerical changes in differentiation remain small. For unlimited storage, when the effect is strongest, higher efficiency reduces optimal differentiation by 5 percentage points, while lower efficiency increases its value by 10 percentage points. For current storage capacities, the difference is less than $1 \%$ for higher efficiency and $3 \%$ for low efficiency.

\section{Concluding remarks}

The ongoing decarbonization of the electricity sector in many countries will substantially increase the share of energy supplied from volatile, intermittent RE sources such as wind and solar. A key challenge, also for bolstering policy support for the decarbonization through more renewables, is to achieve the integration of large amounts of highly volatile generation in electricity markets at moderate costs. Much of the ongoing discussions in both the academic literature and among policy-makers have focused on how increased volumes of electricity storage can serve as a buffering mechanism to cope with market volatility and system integration cost. In light of large uncertainties about the cost, availability, and potential of future storage technologies when deployed at large scales, this paper has examined the suitability of an alternative mechanism for buffering volatility that is based on modifying the design of RE support schemes to take into account the heterogeneous value of different RE technologies in terms of their system integration costs.

To provide a conceptual and empirically-grounded framework for thinking about the economics of integrating high shares of volatile RE sources into an electricity market, we have developed a numerical partial equilibrium model of the wholesale electricity market which resolves output decisions on hourly markets, time-dependent demand and resource availabilities of wind and the sun, investment decisions in production capacity, curtailment decisions to maintain system stability, and a detailed representation of short-term and longer-term electricity storage. The decentralized market model is embedded in a welfare-maximizing problem of a benevolent regulator who chooses RE support policies (through subsidies on RE output which we model as a feed-in premium on top of the market price) in order to implement an electricity market with a high share of intermittent RE at the lowest cost to society. While we have calibrated the model to current market conditions of the German electricity market, we believe that the main insights emerging from our analysis largely carry over to the electricity market context of other countries, too.

Our analysis provides several important insights. First, we find that the storage capacity needed to accommodate a high share of intermittent RE output is relatively moderate, even under a technology-neutral RE support scheme. This implies that the potentially high costs of providing storage at large scale in the future need not jeopardize the achievement of environmental targets (i.e., the reduction of $\mathrm{CO}_{2}$ emissions through increasing the share of low-carbon renewables). Second, we find that the design of a RE policy can have a significant impact on system integration cost as well as storage capacity needs when there are several intermittent renewable technologies with heterogeneous availability patterns of the underlying natural resources (such as wind and solar energy). The smart differentiation of RE subsidies affects investment patterns in a way which can effectively reduce the curtailment of excess generation, in turn lowering the need for costly investment in energy storage. We use a simple cost-benefit framework to show that optimal subsidy differentiation significantly reduces the level of optimal storage. In this sense, concerns about the costs and availability of future storage technologies to be able to integrate a high share of intermittent RE output in electricity markets and to achieve environmental goals are even more diminished if a smart design of RE support policies is chosen. Third, within our modeling framework which captures high RE shares up to $80 \%$ but not a completely decarbonized system, we find that the type of storage most likely needed is short-term to medium-term storage. The additional benefits from long-term seasonal storage are relatively modest and most likely much smaller than its investment costs.

The necessary abstraction and assumptions of the electricity market model imply that several caveats should be kept in mind when interpreting our results. Future costs and benefits of RE and storage technologies are highly uncertain in the present. We therefore make use of current cost estimates in our model calibration and cost-benefit analysis and thus cannot and do not aim to predict exact numbers for future systems. We focus on one policy scheme (a feed-in premium per MWh of renewable energy produced). There are several other possibilities of designing RE subsidies, including a subsidy scheme where the subsidy goes to zero whenever the electricity price reaches zero and a subsidy per MW of installed capacity. We leave the role of technology-differentiation in such alternative policy designs to future 
research. Our findings rely on the assumption of fixed renewable production profiles taken from the base year of our simulation which might not be the best approximation of future profiles. Therefore, we cannot address questions of different predictability of RE technologies and its implications for optimal subsidy differentiation. The marginal benefits curve of storage capacity which we construct does not capture all potential benefits. We focus on the biggest contributor to system integration cost, curtailment of RE generation, but storage will also reduce cost originating from stochastic variation of weather conditions and-if it is organized in smaller decentralized units-storage can also reduce the need for costly transmission grid extensions. At the same time, a greater interconnection via transmission capacities to neighboring markets has the potential to reduce the marginal benefits from storage investment because it permits a more efficient use of existing RE capacities and storage capacities over a larger geographical area. The combined effect on the marginal benefit curve depends on specific details of the electricity market in question and is beyond the scope of this work. The same is true for additional benefits from ancillary services storage could provide (see, e.g. Newbery, 2016, for an evaluation of earnings from ancillary services). We also abstract from complications from natural water inflow that might arise in a system with substantial capacities of large-scale hydro dams. Our generic storage technology does not depend on external weather phenomena but in such a real-world system hydrological constraints could very well interact with intermittency from wind and solar power. These could only be addressed by a more complex model incorporating the hydrological cycles of a specific region which is beyond the scope of this article. Similarly, an extension of the model could explicitly incorporate demand-side management as an alternative form of energy storage. This would require careful modeling of the different types of participants and system costs.

Our analysis should thus not be viewed as a comprehensive costbenefit assessment but rather stresses the point that policy design greatly matters for minimizing the economic cost of achieving $\mathrm{CO}_{2}$ emissions reductions through integrating large amounts of energy supply from carbon-free but volatile RE technologies. While this point has been overlooked so far, it should be taken into account when discussing ways to reduce system integration cost from intermittent $R E$. The potential benefits of subsidy differentiation may also lead the way to re-thinking future RE policy design in terms of specific features of technologies (such as its impact on system stability, interaction with storage or transmission grids) rather than being technologyneutral or tailored to each technology individually. One example for this could be a subsidy for system stability or supply flexibility. We leave an analysis of such policies for future research. With regard to future storage needs, we concur with Zerrahn et al. (2018) who show that the expansion of energy storage capacity will arguably not constitute a limiting factor to integrate large shares of volatile RE supply in electricity markets needed to combat climate change.

\section{References}

50Hertz, 2018. Wind Production in the German Region Controlled by 50 Hertz. http:// www.50hertz.com/en/Grid-Data/Wind-power/Archive-Wind-power.

Abrell, J., Kosch, M., Rausch, S., 2019a. Carbon abatement with renewables: evaluating wind and solar subsidies in Germany and Spain. J. Public Econ. 169, 172-202.

Abrell, J., Rausch, S., Streitberger, C., 2019b. The economics of renewable energy support. J. Public Econ. 176, 94-117.

AEE, 2018. Agentur für Erneuerbare Energien. https://www.foederal-erneuerbar.de/ startseite.
Amprion, 2018. Wind production in the German region controlled by amprion. https:// www.amprion.net/Netzkennzahlen/Windenergieeinspeisung/index-3.html.

BNetzA, 2019. Bundesnetzagentur: Ergebnisse der gemeinsamen Ausschreibungen nach dem EEG. https://www.bundesnetzagentur.de/SharedDocs/ Pressemitteilungen/DE/2019/20190418_Ausschreibungen.html?nn=265778.

Carson, R.T., Novan, K., 2013. The private and social economics of bulk electricity storage. J. Environ. Econ. Manag. 66, 404-423.

Crampes, C., Moreaux, M., 2010. Pumped storage and cost saving. Energy Econ. 32, 325-333. https://EconPapers.repec.org/RePEc:eee:eneeco:v:32:y:2010:i:2:p: 325-333.

Dirkse, S.P., Ferris, M.C., 1995. The path solver: a non-monotone stabilization scheme for mixed complementarity problems. Optim. Methods Softw. 5, 123-156.

Egerer, J., Gerbaulet, C., Ihlenburg, R., Kunz, F., Reinhard, B., von Hirschhausen, C., Weber, A., Weibezahn, J., 2014. Electricity Sector Data for Policy-relevant Modeling: Data Documentation and Applications to the German and European Electricity Markets. . German Institute for Economic Research., Data Documentation 72 DIW Berlin.

ENTSO-E, 2016. Hourly consumption. https://www.entsoe.eu/db-query/consumption/ mhlv-a-specific-country-for-a-specific-month.

European Commission, 2011. Communication from the Commission to the European Parliament, the Council, the European Economic and Social Committee and the Committee of the Regions Energy Roadmap 2050 /* com/2011/0885 final */. https://eur-lex.europa.eu/legal-content/EN/ALL/?uri=CELEX:52011DC0885.

Fell, H., Linn, J., 2013. Renewable electricity policies, heterogeneity, and cost effectiveness. J. Environ. Econ. Manag. 66, 688-707.

Gowrisankaran, G., Reynolds, S.S., Samano, M., 2016. Intermittency and the value of renewable energy. J. Polit. Econ. 124, 1187-1234.

Hartmann, N., Eltrop, L., Bauer, N., et al. 2012. Stromspeicherpotenziale für deutschland (electricity storage potentials for Germany). Institut für Energiewirtschaft und Rationelle Energieanwendung (IER), Institut für Strömungsmechanik und Hydraulische Strömungsmaschinen (IHS), Zentrum für Sonnenenergie-und Wasserstoff-Forschung Baden-Württemberg (ZSW), Universität Stuttgart, Stuttgart, Germany.

Helm, C., Mier, M., 2018. Subsidising renewables but taxing storage? Second-best policies with imperfect carbon pricing. Oldenburg Discussion Papers in Economics, No. V-413-18.

Hirth, L., 2015. Market value of solar power: is photovoltaics cost-competitive? IET Renew. Power Gener. 9, 37-45. https://doi.org/10.1049/iet-rpg.2014.0101.

Hirth, L., Ueckerdt, F., Edenhofer, O., 2015. Integration costs revisited - an economic framework for wind and solar variability. Renew. Energy 74, 925-939.

International Energy Agency, 2018. World energy outlook. Technical Report.

IPCC, 2018. An IPCC special report on the impacts of global warming of $1.5^{\circ} \mathrm{C}$ above pre-industrial levels and related global greenhouse gas emission pathways, in the context of strengthening the global response to the threat of climate change, sustainable development, and efforts to eradicate poverty. https://www.ipcc.ch/ sr15/.

Kost, C., Mayer, J.N., Thomsen, J., Hartmann, N., Senkpiel, C., Philipps, S., Nold, S., Lude, S., Saad, N., Schlegl, T., 2013. Levelized Cost of Electricity: Renewable Energy Technologies. Frauenhofer Institute for Solar Energy Systems ISI Study.

Linn, J., Shih, J.-S., 2016. Does electricity storage innovation reduce greenhouse gas emissions? Discussion Paper 16-37 Resources for the Future. Resources for the future.

Luo, Z.-Q., Pang, J.-S., Ralph, D., 1996. Mathematical Programs With Equilibrium Constraints. Cambridge University Press.

Mathiesen, L., 1985. Computation of economic equilibria by a sequence of linear complementarity problems. Math. Program. Stud. 23, 144-162.

Newbery, D., 2016. A simple introduction to the economics of storage: shifting demand and supply over time and space. EPRG Working Paper. pp. 1626.

Open Power System Data, 2017. Conventional power plants Germany. https://data. open-power-system-data.org/conventional_power_plants/.

Rastler, D., 2010. Electric Energy Storage Technology Options: A Primer on Applications, Costs \& Benefits - An EPRI Executive Summary.

Rutherford, T.F., 1995. Extension of GAMS for complementarity problems arising in applied economics. J. Econ. Dyn. Control. 19, 1299-1324.

Schwab, A.J., 2009. Elektro-Energiesysteme. Springer., pp. 172-175

Sinn, H.-W., 2017. Buffering volatility: a study on the limits of Germany's energy revolution. Eur. Econ. Rev. 99, 130-150.

Tennet, 2018. Wind Production in the German Region Controlled by Tennet.

TransnetBW, 2018. Wind Production in the German Region Controlled by TransnetBW.

Wibulpolprasert, W., 2016. Optimal environmental policies and renewable energy investment: evidence from the texas electricity market. Clim. Chang. Econ. 7.

Zakeri, B., Syri, S., 2015. Electrical energy storage systems: a comparative life cycle cost analysis. Renew. Sust. Energ. Rev. 42, 569-596.

Zerrahn, A., Schill, W.-P., Kemfert, C., 2018. On the economics of electrical storage for variable renewable energy sources. Eur. Econ. Rev. 108, 259-279. 